\title{
PULL-BACKS AND FIBRATIONS IN APPROXIMATE PRO-CATEGORIES
}

\author{
TAKAHISA MiYATA
}

Kobe University, Japan

\begin{abstract}
In this paper we introduce the category Apro- ANR called the approximate pro-category of ANR's, whose objects are all systems of ANR's and whose morphisms are obtained as equivalence classes of system maps for some equivalence relation. We show that any 2-sink $\boldsymbol{X} \stackrel{f}{\longrightarrow} \boldsymbol{Z} \stackrel{g}{\longleftarrow} \boldsymbol{Y}$ in Apro-ANR admits a weak pull-back and it admits a pull-back if they are systems of compact ANR's. Moreover, it admits a pull-back if they are objects of pro- ANRU. Here ANRU is the full subcategory of the category Unif of uniform spaces and uniform maps, whose objects are uniform absolute neighborhood retracts (ANRU's) in the sense of Isbell. We define the approximate homotopy lifting property (AHLP) for morphisms in Apro- ANR and show that the category Apro- ANR with fibration $=$ morphism with the AHLP with respect to paracompact spaces, and weak equivalence $=$ morphism inducing an isomorphisms in pro- $\mathrm{H}(\mathrm{ANR})$ satisfies composition and factorization axioms and part of pull-back axiom for fibration category in the sense of Baues. Finally, we show that the limit of the pull-back of any 2-sink $\boldsymbol{X} \stackrel{f}{\longrightarrow} \boldsymbol{Z} \stackrel{g}{\leftrightarrows} \boldsymbol{Y}$ in Apro- ANR consisting of systems of compact ANR's is a pull-back in the category Top of topological spaces and continuous maps, and conversely every pull-back in the full subcategory $\mathrm{CH}$ of Top whose objects are compact Hausdorff spaces admits an expansion which is a pull-back in Apro- ANR.
\end{abstract}

\section{INTRODUCTION}

Although the category Top of topological spaces and continuous maps has pull-backs, subcategories of Top do not have pull-backs in general. For example, the full subcategory ANR of Top whose objects are ANR's does not

2000 Mathematics Subject Classification. 54C56, 54C55, 55U35.

Key words and phrases. Approximate pro-category, pull-back, approximate homotopy lifting property. 
have pull-backs. However, every 2 -sink $X \stackrel{f}{\longrightarrow} Z \stackrel{g}{\longleftarrow} Y$ in ANR has a pullback in ANR if the map $f$ is a strong fibration [13], i.e., it has the strong homotopy lifting property with respect to any spaces $A$ : if $h: A \rightarrow X$ and $H: A \times I \rightarrow Z$ are maps such that $f h=H_{0}$, there is a map $\tilde{H}: A \times I \rightarrow X$ such that $h=\tilde{H}_{0}$ and $f \tilde{H}=H$, and whenever $H$ is constant on $a \times I, \tilde{H}$ is constant on $a \times I$.

An analogous result holds for the pro-category. A 2-sink $\boldsymbol{X} \stackrel{f}{\longrightarrow} \boldsymbol{Z} \stackrel{g}{ } \boldsymbol{Y}$ in the full subcategory $\mathcal{A N R}$ of pro- Top whose objects are the objects called ANR-objects has a pull-back in $\mathcal{A N \mathcal { R }}$ if the morphism $f$ is a strong profibration [14], i.e., it has the following strong homotopy lifting property with respect to any spaces $A$ : For any admissible pair $(\lambda, \mu) \in \Lambda \times M$ there exists an admissible pair $\left(\lambda^{\prime}, \mu^{\prime}\right) \geq(\lambda, \mu)$ such that whenever $h: A \times 0 \rightarrow X_{\lambda^{\prime}}$ and $H: A \times I \rightarrow Y_{\mu^{\prime}}$ are maps such that $f_{\mu^{\prime}} p_{f\left(\mu^{\prime}\right) \lambda^{\prime}} h=H_{0}$, then there is a map $\tilde{H}: A \times I \rightarrow X_{\lambda}$ satisfying $p_{\lambda \lambda^{\prime}} h=\tilde{H}_{0}$ and $f_{\mu} p_{f(\mu) \lambda} \tilde{H}=q_{\lambda \lambda^{\prime}} H$ and the property that whenever $H$ is constant on $a \times I$, then $\tilde{H}$ is constant on $a \times I$. Here ANR-objects are objects which are isomorphic to systems of ANR's in pro- Top.

The pro-category pro- ANR is a significant category because shape theory is based on the homotopy pro-category pro- $\mathrm{H}($ ANR) of ANR's. However, the category pro- ANR does not have pull-backs in general unless the morphism $f$ has the appropriate homotopy lifting property. In this paper we consider a category which induces the homotopy pro-category pro- H(ANR) but has pull-backs for any 2-sinks in the category. More precisely, we introduce the category Apro-ANR called the approximate pro-category of ANR's, whose objects are all systems of ANR's and whose morphisms are obtained as equivalence classes of system maps for some equivalence relation. We show that any 2 -sink $\boldsymbol{X} \stackrel{f}{\longrightarrow} \boldsymbol{Z} \stackrel{g}{\longleftarrow} \boldsymbol{Y}$ in Apro-ANR has a weak pull-back and it admits a pull-back if they are systems of compact ANR's. More generally, any 2-sink $\boldsymbol{X} \stackrel{f}{\longrightarrow} \boldsymbol{Z} \stackrel{g}{\longleftarrow} \boldsymbol{Y}$ in Apro- ANRU admits a pull-back. Here ANRU is the full subcategory of the category Unif of uniform spaces and uniform maps, whose objects are uniform absolute neighborhood retracts (ANRU's) in the sense of Isbell [5].

Mardešić and Rushing [8] introduced the notion of approximate profibration for morphisms in the pro-category pro- ANR. An approximate profibration is a morphism having the approximate homotopy lifting property with respect to any spaces. Secondly in this paper, we define the approximate homotopy lifting property for morphisms in the approximate pro-category Apro- ANR and show that the category Apro- ANR with fibration = morphism with the AHLP with respect to paracompact spaces, and weak equivalence = morphism inducing an isomorphisms in pro- $\mathrm{H}(\mathrm{ANR})$ satisfies composition and factorization axioms and part of pull-back axiom for fibration category in the sense of Baues [2]. The notion of fibration category was introduced by Baues 
[2], and it makes the constructions of the homotopy theory available in more contexts than the notion of model category by weakening the assumptions and concentrating on fibrations.

Finally, we show that the limit of the pull-back of any 2-sink $\boldsymbol{X} \stackrel{f}{\longrightarrow}$ $\boldsymbol{Z} \stackrel{g}{\longleftarrow} \boldsymbol{Y}$ in Apro- ANR consisting of systems of compact ANR's is a pull-back in the category Top of topological spaces and continuous maps, and conversely every pull-back in the full subcategory $\mathrm{CH}$ of Top whose objects are compact Hausdorff spaces admits an expansion in Apro- ANR which is a pull-back in Apro- ANR.

\section{Approximate PRO-CATEgories}

For any coverings $\mathcal{U}$ and $\mathcal{V}$ of a set $X, \mathcal{U}$ is said to refine $\mathcal{V}$, in notation, $\mathcal{U}<\mathcal{V}$, provided for each $U \in \mathcal{U}$ there is $V \in \mathcal{V}$ such that $U \subseteq V$. Let $\mathcal{U} \wedge \mathcal{V}=\{U \cap V: U \in \mathcal{U}, V \in \mathcal{V}\}$ and $\mathcal{U} \times \mathcal{V}=\{U \times V: U \in \mathcal{U}, V \in \mathcal{V}\}$. Let $\mathcal{U}$ and $\mathcal{V}$ be coverings of sets $X$ and $Y$, respectively. For any subset $A$ of $X$, let $\operatorname{st}(A, \mathcal{U})=\cup\{U \in \mathcal{U}: U \cap A \neq \emptyset\}$ and $\mathcal{U} \mid A=\{U \cap A: U \in \mathcal{U}\}$. If $A=\{x\}$, we write st $(x, \mathcal{U})$ for $\operatorname{st}(\{x\}, \mathcal{U})$. Let $\operatorname{st} \mathcal{U}=\{\operatorname{st}(U, \mathcal{U}): U \in \mathcal{U}\}$. Two points $x, x^{\prime} \in X$ are $\mathcal{U}$-near, denoted $\left(x, x^{\prime}\right)<\mathcal{U}$, provided $x, x^{\prime} \in U$ for some $U \in \mathcal{U}$. Two functions $f, g: X \rightarrow Y$ are $\mathcal{V}$-near, denoted $(f, g)<\mathcal{V}$, provided $(f(x), g(x))<\mathcal{V}$ for each $x \in X$. For any function $f: X \rightarrow Y$, let $f \mathcal{U}=\{f(U): U \in \mathcal{U}\}$ and $f^{-1} \mathcal{V}=\left\{f^{-1}(V): V \in \mathcal{V}\right\}$.

Let $\boldsymbol{X}=\left(X_{\lambda}, p_{\lambda \lambda^{\prime}}, \Lambda\right)$ and $\boldsymbol{Y}=\left(Y_{\mu}, q_{\mu \mu^{\prime}}, M\right)$ be inverse systems (systems, in short) in a category $\mathcal{C}$. A system map $\left(f, f_{\mu}\right): \boldsymbol{X} \rightarrow \boldsymbol{Y}$ consists of a function $f: M \rightarrow \Lambda$ and morphisms $f_{\mu}: X_{f(\mu)} \rightarrow Y_{\mu}$ for $\mu \in M$ such that for $\mu<\mu^{\prime}$ there is $\lambda>f(\mu), f\left(\mu^{\prime}\right)$ such that $f_{\mu} \circ p_{f(\mu) \lambda}=q_{\mu \mu^{\prime}} \circ f_{\mu^{\prime}} \circ p_{f\left(\mu^{\prime}\right) \lambda}$.

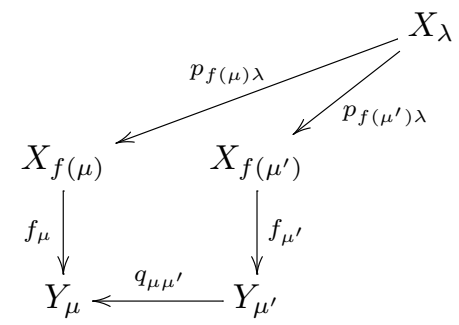

For systems $\boldsymbol{X}=\left(X_{\lambda}, p_{\lambda \lambda^{\prime}}, \Lambda\right)$ and $\boldsymbol{Y}=\left(Y_{\lambda}, q_{\lambda \lambda^{\prime}}, \Lambda\right)$ with the same index set, a level morphism $\left(f_{\lambda}\right): \boldsymbol{X} \rightarrow \boldsymbol{Y}$ consists of morphisms $f_{\lambda}: X_{\lambda} \rightarrow Y_{\lambda}$ for $\lambda \in \Lambda$ in $\mathcal{C}$ such that $f_{\lambda} \circ p_{\lambda \lambda^{\prime}}=q_{\lambda \lambda^{\prime}} \circ f_{\lambda^{\prime}}$ for $\lambda<\lambda^{\prime}$.

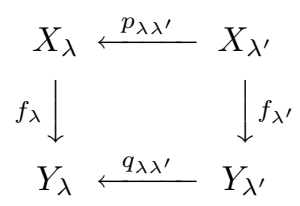


Throughout the paper, we assume space=topological space, map = continuous map, uniform map=uniformly continuous map between uniform spaces. Let Top denote the category of spaces and maps, and let ANR denote the full subcategory of Top whose objects are ANR's.

For any subcategory $\mathcal{C}$ of Top, systems in $\mathcal{C}$ are called $\mathcal{C}$-systems. For any space $X$, let $\operatorname{Cov}(X)$ denote the family of all normal open coverings of $X$. In what follows, we consider the case where $\mathcal{C}$ is any subcategory of Top.

Now we define the category Apro- $\mathcal{C}$, which we call the approximate procategory. The objects of Apro- $\mathcal{C}$ are all $\mathcal{C}$-systems. Morphisms $\boldsymbol{f}: \boldsymbol{X} \rightarrow \boldsymbol{Y}$ are defined as follows: First, we define a relation $\sim_{a}$ between system maps $\boldsymbol{X} \rightarrow \boldsymbol{Y}$ by saying $\left(f, f_{\mu}\right) \sim_{a}\left(g, g_{\mu}\right)$ if and only if for each $\mu \in M$ and $\mathcal{V} \in \operatorname{Cov}\left(Y_{\mu}\right)$ there exists $\lambda>f(\mu), g(\mu)$ such that $\left(f_{\mu} p_{f(\mu) \lambda}, g_{\mu} p_{g(\mu) \lambda}\right)<\mathcal{V}$.

Proposition 2.1. $\sim_{a}$ is an equivalence relation.

Proof. It suffices to verify the transitivity. Suppose that $\left(f, f_{\mu}\right) \sim_{a}$ $\left(g, g_{\mu}\right)$ and $\left(g, g_{\mu}\right) \sim_{a}\left(h, h_{\mu}\right)$. Let $\mu \in M$ and $\mathcal{V} \in \operatorname{Cov}\left(Y_{\mu}\right)$, and take $\mathcal{V}^{\prime} \in$ $\operatorname{Cov}\left(Y_{\mu}\right)$ such that st $\mathcal{V}^{\prime}<\mathcal{V}$. There is $\lambda>f(\mu), g(\mu), h(\mu)$ such that

$$
\begin{aligned}
& \left(f_{\mu} p_{f(\mu) \lambda}, g_{\mu} p_{g(\mu) \lambda}\right)<\mathcal{V}^{\prime}, \\
& \left(g_{\mu} p_{g(\mu) \lambda}, h_{\mu} p_{h(\mu) \lambda}\right)<\mathcal{V}^{\prime} .
\end{aligned}
$$

So, $\left(f_{\mu} p_{f(\mu) \lambda}, h_{\mu} p_{h(\mu) \lambda}\right)<\mathcal{V}$, which means $\left(f, f_{\mu}\right) \sim_{a}\left(h, h_{\mu}\right)$ as required.

We define the morphisms $\boldsymbol{f}: \boldsymbol{X} \rightarrow \boldsymbol{Y}$ in Apro- $\mathcal{C}$ as the equivalence classes of system maps $\left(f, f_{\mu}\right): \boldsymbol{X} \rightarrow \boldsymbol{Y}$.

For any system maps $\left(f, f_{\mu}\right): \boldsymbol{X} \rightarrow \boldsymbol{Y}$ and $\left(g, g_{\nu}\right): \boldsymbol{Y} \rightarrow \boldsymbol{Z}=$ $\left(Z_{\nu}, r_{\nu \nu^{\prime}}, N\right)$, consider the composition $\left(g, g_{\nu}\right) \circ\left(f, f_{\mu}\right)=\left(g_{\nu} f_{g(\nu)}, f g\right): \boldsymbol{X} \rightarrow$ Z.

Proposition 2.2. (1) If $\left(f^{\prime}, f_{\mu}^{\prime}\right): \boldsymbol{X} \rightarrow \boldsymbol{Y}$ is a system map such that $\left(f, f_{\mu}\right) \sim_{a}\left(f^{\prime}, f_{\mu}^{\prime}\right)$, then $\left(g, g_{\nu}\right) \circ\left(f, f_{\mu}\right) \sim_{a}\left(g, g_{\nu}\right) \circ\left(f^{\prime}, f_{\mu}^{\prime}\right)$.

(2) If $\left(g^{\prime}, g_{\nu}^{\prime}\right): \boldsymbol{Y} \rightarrow \boldsymbol{Z}$ is a system map such that $\left(g, g_{\nu}\right) \sim_{a}\left(g^{\prime}, g_{\nu}^{\prime}\right)$, then $\left(g, g_{\nu}\right) \circ\left(f, f_{\mu}\right) \sim_{a}\left(g^{\prime}, g_{\nu}^{\prime}\right) \circ\left(f, f_{\mu}\right)$.

Proof. For assertion (1), let $\nu \in N$, and let $\mathcal{V} \in \operatorname{Cov}\left(Z_{\nu}\right)$. Then there is $\lambda>f g(\nu), f^{\prime} g(\nu)$ such that

$$
\left(f_{g(\nu)} p_{f g(\nu) \lambda}, f_{g(\nu)}^{\prime} p_{f^{\prime} g(\nu) \lambda}\right)<g_{\nu}^{-1} \mathcal{V}
$$

which implies

$$
\left(g_{\nu} f_{g(\nu)} p_{f g(\nu) \lambda}, g_{\nu} f_{g(\nu)}^{\prime} p_{f^{\prime} g(\nu) \lambda}\right)<\mathcal{V}
$$

For assertion (2), again let $\nu \in N$, and let $\mathcal{W} \in \operatorname{Cov}\left(Z_{\nu}\right)$. Then there is $\mu>g(\nu), g^{\prime}(\nu)$ such that

$$
\left(g_{\nu} q_{g(\nu) \mu}, g_{\nu}^{\prime} q_{g^{\prime}(\nu) \mu}\right)<\mathcal{W}
$$


Since $\left(f, f_{\mu}\right)$ is a system map, there is $\lambda>f g(\nu), f g^{\prime}(\nu), f(\mu)$ such that

$$
\begin{aligned}
& f_{g(\nu)} p_{f g(\nu) \lambda}=q_{g(\nu) \mu} f_{\mu} p_{f(\mu) \lambda}, \\
& f_{g^{\prime}(\nu)} p_{f g^{\prime}(\nu) \lambda}=q_{g^{\prime}(\nu) \mu} f_{\mu} p_{f(\mu) \lambda} .
\end{aligned}
$$

Thus

as required.

$$
\left(g_{\nu} f_{g(\nu)} p_{f g(\nu) \lambda}, g_{\nu}^{\prime} f_{g^{\prime}(\nu)} p_{f g^{\prime}(\nu) \lambda}\right)<\mathcal{W}
$$

For any morphisms $\boldsymbol{f}: \boldsymbol{X} \rightarrow \boldsymbol{Y}$ and $\boldsymbol{g}: \boldsymbol{Y} \rightarrow \boldsymbol{Z}$, the composition $\boldsymbol{g} \circ \boldsymbol{f}$ : $\boldsymbol{X} \rightarrow \boldsymbol{Z}$ is defined as the morphism represented by $\left(g, g_{\nu}\right) \circ\left(f, f_{\mu}\right)$, where $\left(f, f_{\mu}\right)$ and $\left(g, g_{\nu}\right)$ are system maps representing $\boldsymbol{f}$ and $\boldsymbol{g}$, respectively. The well-definedness of the composition is guaranteed by Proposition 2.2 above.

Let the identity $1_{\boldsymbol{X}}: \boldsymbol{X} \rightarrow \boldsymbol{X}$ be the morphism induced by the system map $\left(1_{X_{\lambda}}, 1_{\Lambda}\right)$ consisting of the identity maps $1_{X_{\lambda}}: X_{\lambda} \rightarrow X_{\lambda}$ and the identity function $1_{\Lambda}: \Lambda \rightarrow \Lambda$.

It is readily seen that Apro- $\mathcal{C}$ together with the composition and the identity becomes a category. Our primary concern is the case where $\mathcal{C}=$ ANR.

From the construction of Apro- $\mathcal{C}$, there is an obvious functor $F:$ pro- $\mathcal{C} \rightarrow$ Apro- $\mathcal{C}$.

We have the following facts for Apro- $\mathcal{C}$, which are analogous to [9, Theorems 2,3 , Ch. I, §1.3]):

Proposition 2.3. Every system $\boldsymbol{X}$ in Apro- $\mathcal{C}$ indexed by a set $\Lambda$ admits an isomorphic system $\boldsymbol{Y}$ indexed by a directed cofinite ordered set $M$ such that the terms and bonding maps of $\boldsymbol{Y}$ are those of $\boldsymbol{X}$ and $\operatorname{card}(M) \leq \operatorname{card}(\Lambda)$.

Proof. This is obvious by [9, Theorem 2, Ch. I, §1.3] and the existence of the functor $F:$ pro- $\mathcal{C} \rightarrow$ Apro- $\mathcal{C}$.

Proposition 2.4. For any morphism $\boldsymbol{f}: \boldsymbol{X} \rightarrow \boldsymbol{Y}$ in Apro- $\mathcal{C}$ there exist a morphism $\boldsymbol{f}^{\prime}: \boldsymbol{X}^{\prime} \rightarrow \boldsymbol{Y}^{\prime}$ between cofinite systems $\boldsymbol{X}^{\prime}$ and $\boldsymbol{Y}^{\prime}$ and isomorophisms $\boldsymbol{i}: \boldsymbol{X} \rightarrow \boldsymbol{X}^{\prime}$ and $\boldsymbol{j}: \boldsymbol{Y} \rightarrow \boldsymbol{Y}^{\prime}$ such that $\boldsymbol{f}$ is represented by a level morphism, $\boldsymbol{j} \circ \boldsymbol{f}=\boldsymbol{f}^{\prime} \circ \boldsymbol{i}$, and each term and bonding morphism of $\boldsymbol{X}^{\prime}$ and $\boldsymbol{Y}^{\prime}$ is that of $\boldsymbol{X}$ and $\boldsymbol{Y}$, respectively.

Proof. This is proven exactly as for [9, Theorem 3, Ch. I, §1.3], using Proposition 2.3.

For any system map $\left(f, f_{\mu}\right): \boldsymbol{X} \rightarrow \boldsymbol{Y}$, a pair $(\lambda, \mu) \in \Lambda \times M$ is said to be admissible if $\lambda>f(\mu)$.

Proposition 2.5. Let $\boldsymbol{f}: \boldsymbol{X} \rightarrow \boldsymbol{Y}$ be a morphism in Apro- $\mathcal{C}$ which is represented by a system map $\left(f, f_{\mu}\right): \boldsymbol{X} \rightarrow \boldsymbol{Y}$. Then if $\boldsymbol{f}$ is an isomorphism in Apro- $\mathcal{C}$, then for each admissible pair $(\lambda, \mu) \in \Lambda \times M$ and for each $\mathcal{U} \in$ $\operatorname{Cov}\left(X_{\lambda}\right)$ and $\mathcal{V} \in \operatorname{Cov}\left(Y_{\mu}\right)$ there exist an admissible pair $\left(\lambda^{\prime}, \mu^{\prime}\right)>(\lambda, \mu)$ and a map $h: Y_{\mu^{\prime}} \rightarrow X_{\lambda}$ such that 


$$
\begin{aligned}
\left(p_{\lambda \lambda^{\prime}}, h f_{\mu^{\prime}} p_{f\left(\mu^{\prime}\right) \lambda^{\prime}}\right) & <\mathcal{U}, \\
\left(f_{\mu} p_{f(\mu) \lambda} h, q_{\mu \mu^{\prime}}\right) & <\mathcal{V} .
\end{aligned}
$$

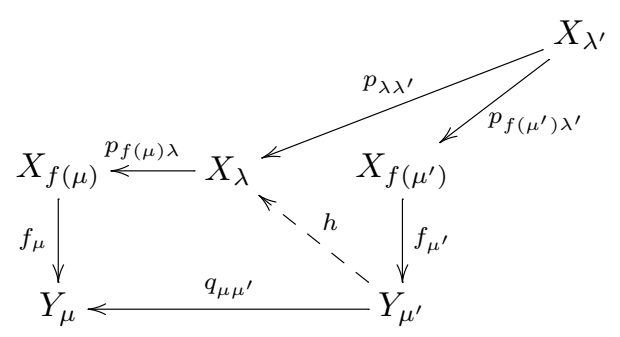

Proof. Let $(\lambda, \mu) \in \Lambda \times M$ be an admissible pair, and let $\mathcal{U} \in \operatorname{Cov}\left(X_{\lambda}\right)$ and $\mathcal{V} \in \operatorname{Cov}\left(Y_{\mu}\right)$. Let $\boldsymbol{g}: \boldsymbol{Y} \rightarrow \boldsymbol{X}$ be a morphism in Apro- $\mathcal{C}$ which is the inverse of $\boldsymbol{f}$, and let $\boldsymbol{g}$ be represented by a system map $\left(g, g_{\lambda}\right)$. Then there exists $\mu^{\prime}>g f(\mu), g(\lambda), \mu$ such that

$$
\begin{aligned}
& \left(f_{\mu} g_{f(\mu)} q_{g f(\mu) \mu^{\prime}}, q_{\mu \mu^{\prime}}\right)<\mathcal{V}, \\
& g_{f(\mu)} q_{g f(\mu) \mu^{\prime}}=p_{f(\mu) \lambda} g_{\lambda} q_{g(\lambda) \mu^{\prime}} .
\end{aligned}
$$

Moreover, there exists $\lambda^{\prime}>\lambda, f g(\lambda), f\left(\mu^{\prime}\right)$ such that

$$
\begin{aligned}
& \left(p_{\lambda \lambda^{\prime}}, g_{\lambda} f_{g(\lambda)} p_{f g(\lambda) \lambda^{\prime}}\right)<\mathcal{U}, \\
& f_{g(\lambda)} p_{f g(\lambda) \lambda^{\prime}}=q_{g(\lambda) \mu^{\prime}} f_{\mu^{\prime}} p_{f\left(\mu^{\prime}\right) \lambda^{\prime}} .
\end{aligned}
$$

Then the map $h=g_{\lambda} q_{g(\lambda) \mu^{\prime}}$ satisfies (2.1) and (2.2) as required.

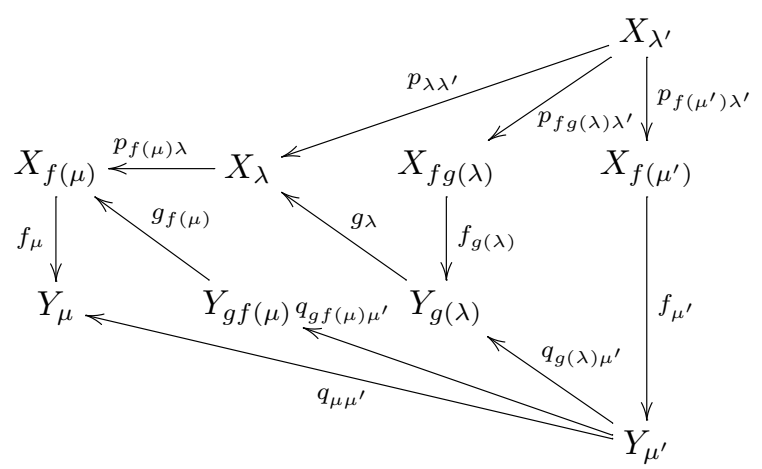

A system map $\boldsymbol{p}=\left(p_{\lambda}\right): X \rightarrow \boldsymbol{X}=\left(X_{\lambda}, p_{\lambda \lambda^{\prime}}, \Lambda\right)$ is a resolution of $X$ if it satisfies the following two conditions:

(R1) For each ANR $P, \mathcal{V} \in \operatorname{Cov}(P)$ and map $f: X \rightarrow P$, there exist $\lambda \in \Lambda$ and a map $g: X_{\lambda} \rightarrow P$ such that $\left(g p_{\lambda}, f\right)<\mathcal{V}$, and 
(R2) For each ANR $P$ and $\mathcal{V} \in \operatorname{Cov}(P)$, there exists $\mathcal{V}^{\prime} \in \operatorname{Cov}(P)$ such that whenever $\lambda \in \Lambda$ and $g, g^{\prime}: X_{\lambda} \rightarrow P$ are maps with $\left(g p_{\lambda}, g^{\prime} p_{\lambda}\right)<\mathcal{V}^{\prime}$, then $\left(g p_{\lambda \lambda^{\prime}}, g^{\prime} p_{\lambda \lambda^{\prime}}\right)<\mathcal{V}$ for some $\lambda^{\prime}>\lambda$.

For any subcategory $\mathcal{C}$ of Top, a resolution $\boldsymbol{p}=\left(p_{\lambda}\right): X \rightarrow \boldsymbol{X}=$ $\left(X_{\lambda}, p_{\lambda \lambda^{\prime}}, \Lambda\right)$ is called a $\mathcal{C}$-resolution if all the coordinate spaces $X_{\lambda}$ are in $\mathcal{C}$.

The following is a useful characterization:

Theorem 2.6. ([9, Theorem 5, Ch. I, §. 6.2], [7, §. 5]) A system map $\boldsymbol{p}=\left(p_{\lambda}\right): X \rightarrow \boldsymbol{X}=\left(X_{\lambda}, p_{\lambda \lambda^{\prime}}, \Lambda\right)$ with all $X_{\lambda}$ being ANR's is a resolution of $X$ if and only if it satisfies the following two conditions:

(B1) For each $\lambda \in \Lambda$ and for each open set $U$ in $X_{\lambda}$ such that $\overline{p_{\lambda}(X)} \subseteq U$, there exists $\lambda^{\prime} \geq \lambda$ such that $p_{\lambda \lambda^{\prime}}\left(X_{\lambda^{\prime}}\right) \subseteq U$, and

(B2) For each $\mathcal{U} \in \operatorname{Cov}(X)$, there exist $\lambda \in \Lambda$ and $\mathcal{V} \in \operatorname{Cov}\left(X_{\lambda}\right)$ such that $p_{\lambda}^{-1} \mathcal{V}<\mathcal{U}$

Note here that condition (B1) is equivalent to condition (B1)* below, which was introduced as condition (B4) in [17, 3.5]:

(B1)* For each $\lambda \in \Lambda$ and $\mathcal{U} \in \operatorname{Cov}\left(X_{\lambda}\right)$, there exists $\lambda^{\prime}>\lambda$ such that $p_{\lambda \lambda^{\prime}}\left(X_{\lambda^{\prime}}\right) \subseteq \operatorname{st}\left(p_{\lambda}(X), \mathcal{U}\right)$.

If $\boldsymbol{p}=\left(p_{\lambda}\right): X \rightarrow \boldsymbol{X}=\left(X_{\lambda}, p_{\lambda \lambda^{\prime}}, \Lambda\right)$ is a resolution of a topologically complete space $X$ and if all $X_{\lambda}$ are normal, then $\boldsymbol{p}$ is an inverse limit of $\boldsymbol{X}[9$, Theorem 6 , Ch. I, $\S 6.3]$. Conversely, if $X_{\lambda}$ are compact Hausdorff spaces, then every inverse limit $\boldsymbol{p}: X \rightarrow \boldsymbol{X}$ of $\boldsymbol{X}$ is a resolution of $X[9$, Theorems 4,5 , Ch. I, $\S 5.2]$.

Proposition 2.7. If $\boldsymbol{p}=\left(p_{\lambda}\right): X \rightarrow \boldsymbol{X}=\left(X_{\lambda}, p_{\lambda \lambda^{\prime}}, \Lambda\right)$ is a resolution and if $\left(f, f_{\mu}\right): \boldsymbol{X} \rightarrow \boldsymbol{Y}$ is a system map which induces an isomorphism in Apro- Top, then the system map $\left(f_{\mu} p_{f(\mu)}\right): X \rightarrow \boldsymbol{Y}$ is a resolution.

Proof. We show that the system map $\left(f_{\mu} p_{f(\mu)}\right)$ has properties (R1) and (R2). For (R1), let $P$ be an $\mathrm{ANR}, \mathcal{W} \in \operatorname{Cov}(P)$, and let $h: X \rightarrow P$ be a map. Fix $\mu \in M$, and choose $\mathcal{W}^{\prime} \in \operatorname{Cov}(P)$ such that st $\mathcal{W}^{\prime}<\mathcal{W}$. Then property (R1) for $\left(p_{\lambda}\right)$ implies that there exist $\lambda \geq f(\mu)$ and a map $k: X_{\lambda} \rightarrow P$ such that

$$
\left(h, k p_{\lambda}\right)<\mathcal{W}^{\prime}
$$

By Proposition 2.5 we have an admissible pair $\left(\lambda^{\prime}, \mu^{\prime}\right)>(\lambda, \mu)$ and a map $g: Y_{\mu^{\prime}} \rightarrow X_{\lambda}$ such that

$$
\left(p_{\lambda \lambda^{\prime}}, g f_{\mu^{\prime}} p_{f\left(\mu^{\prime}\right) \lambda^{\prime}}\right)<k^{-1} \mathcal{W}^{\prime}
$$


(2.3) and (2.4) imply $\left(h, k g f_{\mu^{\prime}} p_{f\left(\mu^{\prime}\right) \lambda^{\prime}}<\mathcal{W}\right.$. So, $\left(h, k g f_{\mu^{\prime}} p_{f\left(\mu^{\prime}\right)}\right)<\mathcal{W}$. This verifies property $(\mathrm{R} 1)$.

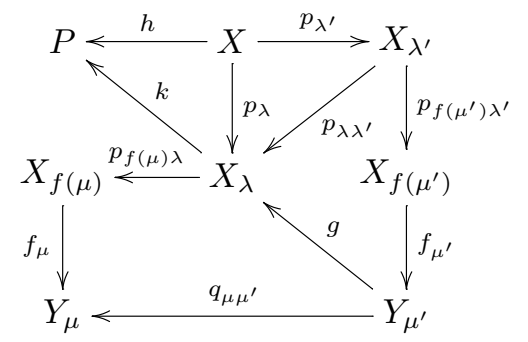

For (R2), let $P$ be an ANR and $\mathcal{W} \in \operatorname{Cov}(P)$. Let $\mathcal{W}_{1} \in \operatorname{Cov}(P)$ be such that st $\mathcal{W}_{1}<\mathcal{W}$. Choose $\mathcal{W}^{\prime} \in \operatorname{Cov}(P)$ with the property that if $\lambda \in \Lambda$ and $h, h^{\prime}: X_{\lambda} \rightarrow P$ are maps such that $\left(h p_{\lambda}, h^{\prime} p_{\lambda}\right)<\mathcal{W}^{\prime}$, then there exists $\lambda^{\prime}>\lambda$ such that $\left(h p_{\lambda \lambda^{\prime}}, h^{\prime} p_{\lambda \lambda^{\prime}}\right)<\mathcal{W}_{1}$. Let $\mu \in M$, and let $h, h^{\prime}: Y_{\mu} \rightarrow P$ be maps such that

$$
\left(h f_{\mu} p_{f(\mu)}, h^{\prime} f_{\mu} p_{f(\mu)}\right)<\mathcal{W}^{\prime} .
$$

Then if we choose $\lambda>f(\mu)$, then

$$
\left(h f_{\mu} p_{f(\mu) \lambda} p_{\lambda}, h^{\prime} f_{\mu} p_{f(\mu) \lambda} p_{\lambda}\right)<\mathcal{W}^{\prime},
$$

so there exists $\lambda^{\prime \prime}>\lambda$ such that

$$
\left(h f_{\mu} p_{f(\mu) \lambda^{\prime \prime}}, h^{\prime} f_{\mu} p_{f(\mu) \lambda^{\prime \prime}}\right)<\mathcal{W}_{1} .
$$

Then by Proposition 2.5, there exist an admissible pair $\left(\lambda^{\prime}, \mu^{\prime}\right)>\left(\lambda^{\prime \prime}, \mu\right)$ and a map $g: Y_{\mu^{\prime}} \rightarrow X_{\lambda^{\prime \prime}}$ such that

$$
\left(f_{\mu} p_{f(\mu) \lambda^{\prime \prime}} g, q_{\mu \mu^{\prime}}\right)<h^{-1} \mathcal{W}_{1} \wedge h^{\prime-1} \mathcal{W}_{1}
$$

By (2.5) and (2.6),

$$
\left(h q_{\mu \mu^{\prime}}, h^{\prime} q_{\mu \mu^{\prime}}\right)<\operatorname{st} \mathcal{W}_{1}<\mathcal{W}
$$

as required.

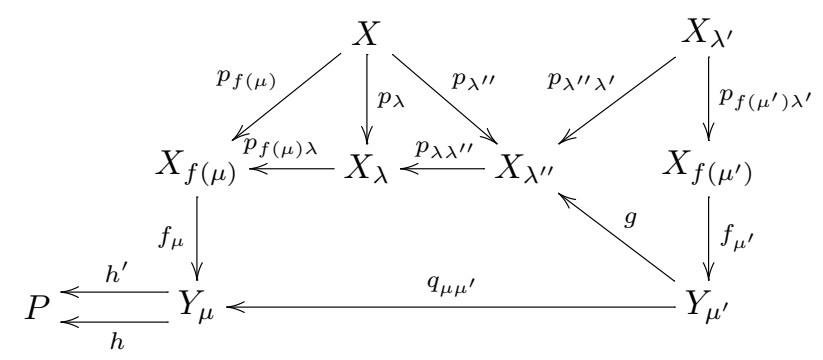




\section{Approximate Systems AND APPROXimate maps}

Let us recall the definitions of approximate system and approximate map in the sense of Mardešić and Watanabe [10]. But we only need the commutative case for our purpose.

A commutative approximate system $\mathfrak{X}=\left(X_{\lambda}, \mathcal{U}_{\lambda}, p_{\lambda \lambda^{\prime}}, \Lambda\right)$ consists of an inverse system $\left(X_{\lambda}, p_{\lambda \lambda^{\prime}}, \Lambda\right)$ and $\mathcal{U}_{\lambda} \in \operatorname{Cov}\left(X_{\lambda}\right), \lambda \in \Lambda$, with the following property:

(AI) For each $\lambda \in \Lambda$ and $\mathcal{U} \in \operatorname{Cov}\left(X_{\lambda}\right)$ there exists $\lambda^{\prime}>\lambda$ such that $\mathcal{U}_{\lambda^{\prime \prime}}<$ $p_{\lambda \lambda^{\prime \prime}}^{-1} \mathcal{U}$ for $\lambda^{\prime \prime}>\lambda^{\prime}$.

If each $\mathcal{U}_{\lambda}$ is the open covering by open $\varepsilon_{\lambda}$-balls for some $\varepsilon_{\lambda}>0$, then we write $\mathfrak{X}=\left(X_{\lambda}, \varepsilon_{\lambda}, p_{\lambda \lambda^{\prime}}, \Lambda\right)$.

For commutative approximate systems $\mathfrak{X}=\left(X_{\lambda}, \mathcal{U}_{\lambda}, p_{\lambda \lambda^{\prime}}, \Lambda\right)$ and $\mathfrak{Y}=$ $\left(Y_{\mu}, \mathcal{V}_{\mu}, q_{\mu \mu^{\prime}}, M\right)$, an approximate map $\left(f, f_{\mu}\right): \mathfrak{X} \rightarrow \mathfrak{Y}$ consists of a function $f: M \rightarrow \Lambda$ and maps $f_{\mu}: X_{f(\mu)} \rightarrow Y_{\mu}, \mu \in M$, with the following property:

(AM) For any $\mu, \mu^{\prime} \in M$ with $\mu<\mu^{\prime}$, there exists $\lambda \in \Lambda, \lambda>f(\mu), f\left(\mu^{\prime}\right)$ such that

$$
\left(q_{\mu \mu^{\prime}} f_{\mu^{\prime}} p_{f\left(\mu^{\prime}\right) \lambda^{\prime}}, f_{\mu} p_{f(\mu) \lambda^{\prime}}\right)<\operatorname{st} \mathcal{V}_{\mu} \text { for } \lambda^{\prime}>\lambda
$$

An approximate map $\left(f, f_{\mu}\right)$ is said to be commutative if

$$
q_{\mu \mu^{\prime}} f_{\mu^{\prime}} p_{f\left(\mu^{\prime}\right) \lambda^{\prime}}=f_{\mu} p_{f(\mu) \lambda^{\prime}} \text { for } \lambda^{\prime}>\lambda
$$

instead of (3.1).

There is another way to express the category Apro- $\mathcal{C}$ using the theory of approximate resolutions. In [10] it is shown that there is an equivalence between the category CTop of topologically complete spaces and maps and the category APRES $\mathrm{ANR}$ of cofinite approximate ANR-resolutions and equivalence classes of approximate maps. The equivalence relation $\sim$ between approximate maps is defined by saying $\left(f, f_{\mu}\right) \sim\left(g, g_{\mu}\right)$ if and only if there exist a finite collection of approximate maps $\left(f_{i},\left(f_{i}\right)_{\mu}\right), i=0,1, \ldots, n$, such that $\left(f_{0},\left(f_{0}\right)_{\mu}\right)=\left(f, f_{\mu}\right),\left(f_{n},\left(f_{n}\right)_{\mu}\right)=\left(g, g_{\mu}\right)$, and $\left(f_{i},\left(f_{i}\right)_{\mu}\right) \equiv$ $\left(f_{i+1},\left(f_{i+1}\right)_{\mu}\right)$. Here for any approximate systems $\mathfrak{X}=\left(X_{\lambda}, \mathcal{U}_{\lambda}, p_{\lambda \lambda^{\prime}}, \Lambda\right)$ and $\mathfrak{Y}=\left(Y_{\mu}, \mathcal{V}_{\mu}, q_{\mu \mu^{\prime}}, M\right)$ and for any two approximate maps $\left(f, f_{\mu}\right),\left(g, g_{\mu}\right): \mathfrak{X} \rightarrow$ $\mathfrak{Y},\left(f, f_{\mu}\right) \equiv\left(g, g_{\mu}\right)$ means that for each $\mu \in M$ there exists $\lambda>f(\mu), g(\mu)$ such that $\left(f_{\mu} p_{f(\mu) \lambda^{\prime}}, g_{\mu} p_{g(\mu) \lambda^{\prime}}\right)<\operatorname{st} \mathcal{V}_{\mu}$ for $\lambda^{\prime}>\lambda$.

Proposition 3.1. For any commutative approximate systems $\mathfrak{X}=$ $\left(X_{\lambda}, \mathcal{U}_{\lambda}, p_{\lambda \lambda^{\prime}}, \Lambda\right)$ and $\mathfrak{Y}=\left(Y_{\mu}, \mathcal{V}_{\mu}, q_{\mu \mu^{\prime}}, M\right)$ in ANR, let $\boldsymbol{X}=\left(X_{\lambda}, p_{\lambda \lambda^{\prime}}, \Lambda\right)$ and $\boldsymbol{Y}=\left(Y_{\mu}, q_{\mu \mu^{\prime}}, M\right)$ be the induced ANR-systems. Then for any system maps $\left(f, f_{\mu}\right),\left(g, g_{\mu}\right): \boldsymbol{X} \rightarrow \boldsymbol{Y},\left(f, f_{\mu}\right) \sim_{a}\left(g, g_{\mu}\right)$ in our sense if and only if $\left(f, f_{\mu}\right) \equiv\left(g, g_{\mu}\right)$ in the sense of [10], considering $\left(f, f_{\mu}\right)$ and $\left(g, g_{\mu}\right)$ as commutative approximate maps $\mathfrak{X} \rightarrow \mathfrak{Y}$. 
Proof. It is obvious that $\left(f, f_{\mu}\right) \sim_{a}\left(g, g_{\mu}\right)$ implies $\left(f, f_{\mu}\right) \equiv\left(g, g_{\mu}\right)$. Conversely, suppose $\left(f, f_{\mu}\right) \equiv\left(g, g_{\mu}\right)$. Let $\mu \in M$, and let $\mathcal{V} \in \operatorname{Cov}\left(Y_{\mu}\right)$. Choose $\mathcal{V}^{\prime} \in \operatorname{Cov}\left(Y_{\mu}\right)$ such that st $\mathcal{V}^{\prime}<\mathcal{V}$, and take $\mu^{\prime}>\mu$ such that $\mathcal{V}_{\mu^{\prime}}<$ $q_{\mu \mu^{\prime}}^{-1} \mathcal{V}^{\prime}$. Note that st $\mathcal{V}_{\mu^{\prime}}<q_{\mu \mu^{\prime}}^{-1}$ st $\mathcal{V}^{\prime}$. By $\left(f, f_{\mu}\right) \equiv\left(g, g_{\mu}\right)$ and the fact that $\left(f, f_{\mu}\right)$ and $\left(g, g_{\mu}\right)$ are system maps, there is $\lambda>f\left(\mu^{\prime}\right), g\left(\mu^{\prime}\right)$ such that

$$
\begin{aligned}
& \left(f_{\mu^{\prime}} p_{f\left(\mu^{\prime}\right) \lambda}, g_{\mu^{\prime}} p_{g\left(\mu^{\prime}\right) \lambda}\right)<\operatorname{st} \mathcal{V}_{\mu^{\prime}}, \\
& f_{\mu} p_{f(\mu) \lambda}=q_{\mu \mu^{\prime}} f_{\mu^{\prime}} p_{f\left(\mu^{\prime}\right) \lambda}, \\
& g_{\mu} p_{g(\mu) \lambda}=q_{\mu \mu^{\prime}} g_{\mu^{\prime}} p_{g\left(\mu^{\prime}\right) \lambda} .
\end{aligned}
$$

Then $\left(q_{\mu \mu^{\prime}} f_{\mu^{\prime}} p_{f\left(\mu^{\prime}\right) \lambda}, q_{\mu \mu^{\prime}} g_{\mu^{\prime}} p_{g\left(\mu^{\prime}\right) \lambda}\right)<\mathcal{V}$, so $\left(f_{\mu} p_{f(\mu) \lambda}, g_{\mu} p_{g(\mu) \lambda}\right)<\mathcal{V}$, which means $\left(f, f_{\mu}\right) \sim_{a}\left(g, g_{\mu}\right)$.

We also need the following result for the later sections.

Proposition 3.2. (1) Every system $\boldsymbol{X}=\left(X_{\lambda}, p_{\lambda \lambda^{\prime}}, \Lambda\right)$ in Top admits a commutative approximate system $\mathfrak{X}=\left(\bar{X}_{\alpha}, \mathcal{U}_{\alpha}, \bar{p}_{\alpha \alpha^{\prime}}, \bar{\Lambda}\right)$ and an increasing function $\sigma_{\boldsymbol{X}}: \bar{\Lambda} \rightarrow \Lambda$ with the following properties:

(a) $\bar{\Lambda}$ is cofinite, directed, and antisymmetric,

(b) $\bar{X}_{\alpha}=X_{\sigma_{\boldsymbol{X}}(\alpha)}$ for $\alpha \in \bar{\Lambda}$, and $\bar{p}_{\alpha \alpha^{\prime}}=p_{\sigma_{\boldsymbol{X}}(\alpha) \sigma_{\boldsymbol{X}}\left(\alpha^{\prime}\right)}$ for $\alpha<\alpha^{\prime}$,

(c) $\mathcal{U}_{\alpha^{\prime}}<\bar{p}_{\alpha \alpha^{\prime}}^{-1} \mathcal{U}_{\alpha}$ for $\alpha<\alpha^{\prime}$,

(d) for any $\lambda \in \Lambda$ and for any $\mathcal{U} \in \operatorname{Cov}\left(X_{\lambda}\right)$ there exists $\alpha \in \bar{\Lambda}$ such that $\sigma_{\boldsymbol{X}}(\alpha)=\lambda$ and $\mathcal{U}_{\alpha}=\mathcal{U}$,

(e) the system map $\left(\sigma_{\boldsymbol{X}}, 1_{\sigma_{\boldsymbol{X}}(\alpha)}\right): \boldsymbol{X} \rightarrow \overline{\boldsymbol{X}}=\left(\bar{X}_{\alpha}, \bar{p}_{\alpha \alpha^{\prime}}, \bar{\Lambda}\right)$ represents an isomorphism in pro- Top.

(2) Every system map $\left(f, f_{\mu}\right): \boldsymbol{X} \rightarrow \boldsymbol{Y}=\left(Y_{\mu}, q_{\mu \mu^{\prime}}, M\right)$ with $f$ being an increasing function admits a commutative approximate map $\left(\bar{f}, \bar{f}_{\beta}\right)$ : $\mathfrak{X} \rightarrow \mathfrak{Y}=\left(\bar{Y}_{\beta}, \mathcal{V}_{\beta}, \bar{q}_{\beta \beta^{\prime}}, \bar{M}\right)$ such that

(a) $\bar{f}$ is an increasing function such that $\sigma_{\boldsymbol{X}} \bar{f}(\beta) \geq f\left(\sigma_{\boldsymbol{Y}}(\beta)\right)$ for $\beta \in \bar{M}$,

(b) $\bar{f}_{\beta}=f_{\sigma_{\boldsymbol{Y}}(\beta)} p_{f \sigma_{\boldsymbol{Y}}(\beta), \sigma_{\boldsymbol{X}} \bar{f}(\beta)}: \bar{X}_{\bar{f}(\beta)} \rightarrow \bar{Y}_{\beta}$,

(c) $\mathcal{U}_{\bar{f}(\beta)}<\bar{f}_{\beta}^{-1} \mathcal{V}_{\beta}$ for $\beta \in \bar{M}$,

(d) the following diagram commutes for $\beta \in \bar{M}$ :

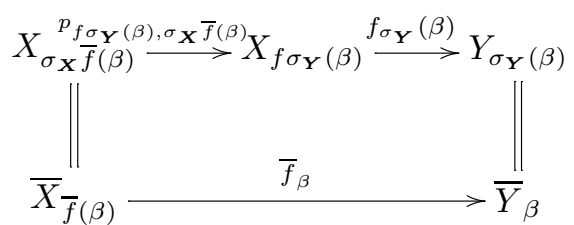

(3) If $\boldsymbol{X}$ is a system in the full subcategory $\mathrm{CM}$ of Top whose objects are compact metric spaces, then we can choose the open coverings $\mathcal{U}_{\alpha}$ in $\mathfrak{X}$ as finite open coverings by open $\varepsilon_{\alpha}$-balls for some $\varepsilon_{\alpha}>0$ such that $\mathrm{d}\left(x, x^{\prime}\right) \leq \varepsilon_{\alpha^{\prime}}$ for $x, x^{\prime} \in \bar{X}_{\alpha^{\prime}}$ implies $\mathrm{d}\left(\bar{p}_{\alpha \alpha^{\prime}}(x), \bar{p}_{\alpha \alpha^{\prime}}\left(x^{\prime}\right)\right)<\varepsilon_{\alpha}$. 
Proof. To show the first assertion, we first recall the definition of the approximate system $\mathfrak{X}$ in the proof of [17, Proposition 3.7]. Let

$$
F(\Lambda)=\left\{(\lambda, \mathcal{U}): \lambda \in \Lambda, \mathcal{U} \in \operatorname{Cov}\left(X_{\lambda}\right)\right\}
$$

and

$$
K(\Lambda)=\{K \subseteq F(\Lambda): K \text { is finite and } K \neq \emptyset\} .
$$

Let $\bar{\Lambda}=K(\Lambda)$ be ordered by inclusion. Then $\bar{\Lambda}$ has property (a). Let $\tau_{\boldsymbol{X}}$ : $\bar{\Lambda} \rightarrow \Lambda$ be a function such that $\tau_{\boldsymbol{X}}(\{(\lambda, \mathcal{U})\})=\lambda$ for $(\lambda, \mathcal{U}) \in F(\Lambda)$. Then there exists an increasing function $\sigma_{\boldsymbol{X}}: \bar{\Lambda} \rightarrow \Lambda$ such that $\sigma_{\boldsymbol{X}} \geq \tau_{\boldsymbol{X}}$ and $\sigma_{\boldsymbol{X}}(\{(\lambda, \mathcal{U})\})=\lambda$ for $(\lambda, \mathcal{U}) \in F(\Lambda)$. As in (b), let $\bar{X}_{\alpha}=X_{\sigma_{\boldsymbol{X}}(\alpha)}$ for $\alpha \in \bar{\Lambda}$ and $\bar{p}_{\alpha \alpha^{\prime}}=p_{\sigma_{X}(\alpha) \sigma_{X}\left(\alpha^{\prime}\right)}$ for $\alpha<\alpha^{\prime}$. For each $\alpha=\left\{\left(\lambda_{1}, \mathcal{U}_{1}\right), \ldots,\left(\lambda_{n}, \mathcal{U}_{n}\right)\right\} \in$ $\bar{\Lambda}$, let $\mathcal{U}_{\alpha}=p_{\lambda_{1} \sigma_{\boldsymbol{X}}(\alpha)}^{-1} \mathcal{U}_{1} \wedge \cdots \wedge p_{\lambda_{n} \sigma_{\boldsymbol{X}}(\alpha)}^{-1} \mathcal{U}_{n} \in \operatorname{Cov}\left(X_{\sigma_{\boldsymbol{X}}(\alpha)}\right)$. The construction immediately implies conditions (c), (d), and (e). This shows the first assertion.

To show the second assertion, let $\left(f, f_{\mu}\right): \boldsymbol{X} \rightarrow \boldsymbol{Y}=\left(Y_{\mu}, q_{\mu \mu^{\prime}}, M\right)$ be a system map. For each $\beta=\left\{\left(\mu_{1}, \mathcal{V}_{1}\right), \ldots,\left(\mu_{n}, \mathcal{V}_{n}\right)\right\} \in \bar{M}$, choose $\mathcal{U} \in \operatorname{Cov}\left(X_{\left.f_{\sigma_{\boldsymbol{Y}}(\beta)}\right)}\right)$ such that $\mathcal{U}<f_{\sigma_{\boldsymbol{Y}}(\beta)}^{-1}\left(q_{\mu_{1} \sigma_{\boldsymbol{Y}}(\beta)}^{-1} \mathcal{V}_{1} \wedge \cdots \wedge q_{\mu_{n} \sigma_{\boldsymbol{Y}}(\beta)}^{-1} \mathcal{V}_{n}\right)$. Then there is an increasing function $\bar{f}: \bar{M} \rightarrow \bar{\Lambda}$ such that $\bar{f}(\beta) \geq\left\{\left(f \sigma_{\boldsymbol{Y}}(\beta), \mathcal{U}\right)\right\}$ for $\beta \in \bar{M}$, and we define a map $\bar{f}_{\beta}: \bar{X}_{\bar{f}(\beta)} \rightarrow \bar{Y}_{\beta}$ by $\bar{f}_{\beta}=f_{\sigma_{\boldsymbol{Y}}(\beta)} p_{f \sigma_{\boldsymbol{Y}}(\beta), \sigma_{\mathbf{X}} \bar{f}(\beta)}$. Thus conditions (a), (b), and (c) are fulfilled. For $\beta<\beta^{\prime}, \sigma_{\boldsymbol{X}} \bar{f}(\beta)<\sigma_{\boldsymbol{X}} \bar{f}\left(\beta^{\prime}\right)$ since $\sigma_{\boldsymbol{X}}$ and $\bar{f}$ are increasing functions. So, we have $\bar{q}_{\beta \beta^{\prime}} \bar{f}_{\beta^{\prime}}=\bar{f}_{\beta} \bar{p}_{\bar{f}(\beta) \bar{f}\left(\beta^{\prime}\right)}$. Thus we have a system map $\left(\bar{f}, \bar{f}_{\beta}\right): \overline{\boldsymbol{X}} \rightarrow \overline{\boldsymbol{Y}}=\left(\bar{Y}_{\beta}, \bar{q}_{\beta \beta^{\prime}}, \bar{M}\right)$, and by the construction we have the commutative diagram (3.2). This proves the second assertion.

The third assertion follows from the compactness of the terms and the cofiniteness of $\bar{\Lambda}$.

\section{Approximate homotopy Lifting PROPERTy}

First, we recall the definition of the approximate homotopy lifting property for system maps [7]. Let $\boldsymbol{X}=\left(X_{\lambda}, p_{\lambda \lambda^{\prime}}, \Lambda\right)$ and $\boldsymbol{Y}=\left(Y_{\mu}, q_{\mu \mu^{\prime}}, M\right)$ be ANR-systems. A system map $\left(f, f_{\mu}\right): \boldsymbol{X} \rightarrow \boldsymbol{Y}$ has the approximate homotoy lifting property (AHLP) with respect to a space $Z$ provided it satisfies the following property:

(AHLP) For any admissible pair $(\lambda, \mu) \in \Lambda \times M$ and for any $\mathcal{U} \in \operatorname{Cov}\left(X_{\lambda}\right)$ and $\mathcal{V} \in \operatorname{Cov}\left(Y_{\mu}\right)$ there exist an admissible pair $\left(\lambda^{\prime}, \mu^{\prime}\right) \geq(\lambda, \mu)$ and $\mathcal{V}^{\prime} \in \operatorname{Cov}\left(Y_{\mu^{\prime}}\right)$ such that whenever $h: Z \times 0 \rightarrow X_{\lambda^{\prime}}$ and $H: Z \times I \rightarrow Y_{\mu^{\prime}}$ are maps with

$$
\left(f_{\mu^{\prime}} p_{f\left(\mu^{\prime}\right) \lambda^{\prime}} h, H_{0}\right)<\mathcal{V}^{\prime}
$$

there exists a map $\tilde{H}: Z \times I \rightarrow X_{\lambda}$ such that

$$
\left(p_{\lambda \lambda^{\prime}}, h, \tilde{H}_{0}\right)<\mathcal{U}
$$




$$
\left(f_{\mu} p_{f(\mu) \lambda} \tilde{H}, q_{\mu \mu^{\prime}} H\right)<\mathcal{V} .
$$

Here $I$ denotes the closed interval $[0,1]$.

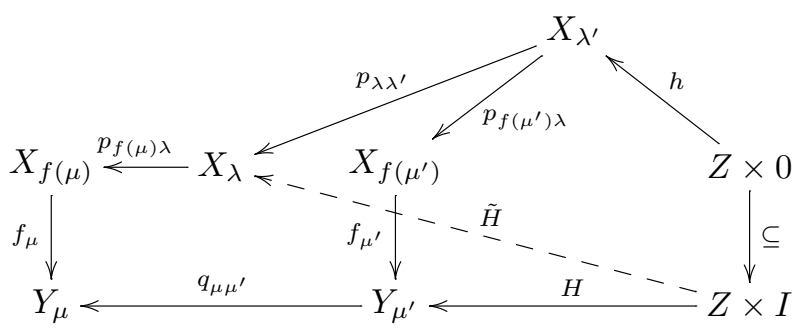

The admissible pair $\left(\lambda^{\prime}, \mu^{\prime}\right)$ and $\mathcal{V}^{\prime}$ in (AHLP) are called a lifting index and lifting mesh, respectively.

The next step is to extend the definition of the AHLP for system maps over morphisms in Apro- ANR.

Proposition 4.1. Let $\left(f, f_{\mu}\right),\left(g, g_{\mu}\right): \boldsymbol{X} \rightarrow \boldsymbol{Y}$ be system maps. If $\left(f, f_{\mu}\right) \sim_{a}\left(g, g_{\mu}\right)$ and if $\left(f, f_{\mu}\right)$ has the AHLP with respect to a space $Z$, so does $\left(g, g_{\mu}\right)$.

Proof. Let $(\lambda, \mu) \in \Lambda \times M$ be an admissible pair for $\left(g, g_{\mu}\right)$, and let $\mathcal{U} \in \operatorname{Cov}\left(X_{\lambda}\right)$ and $\mathcal{V} \in \operatorname{Cov}\left(Y_{\mu}\right)$. Choose $\mathcal{V}_{1} \in \operatorname{Cov}\left(Y_{\mu}\right)$ such that st $\mathcal{V}_{1}<\mathcal{V}$. By $\left(f, f_{\mu}\right) \sim_{a}\left(g, g_{\mu}\right)$, there is $\lambda_{1}>\lambda, f(\mu)$ such that

$$
\left(f_{\mu} p_{f(\mu) \lambda_{1}}, g_{\mu} p_{g(\mu) \lambda_{1}}\right)<\mathcal{V}_{1} .
$$

Apply the AHLP for the system map $\left(f, f_{\mu}\right)$ with the admissible pair $\left(\lambda_{1}, \mu\right)$ and the open coverings $p_{\lambda \lambda_{1}}^{-1} \mathcal{U}$ and $\mathcal{V}_{1}$, and we obtain an admissible pair $\left(\lambda^{\prime}, \mu^{\prime}\right) \geq\left(\lambda_{1}, \mu\right)$ and $\mathcal{V}^{\prime} \in \operatorname{Cov}\left(Y_{\mu^{\prime}}\right)$ with property (AHLP). Choose $\mathcal{V}_{1}^{\prime} \in \operatorname{Cov}\left(Y_{\mu^{\prime}}\right)$ such that st $\mathcal{V}_{1}^{\prime}<\mathcal{V}^{\prime}$. Again by $\left(f, f_{\mu}\right) \sim_{a}\left(g, g_{\mu}\right)$, there is $\lambda_{1}^{\prime}>\lambda^{\prime}, g\left(\mu^{\prime}\right)$ such that

$$
\left(f_{\mu^{\prime}} p_{f\left(\mu^{\prime}\right) \lambda_{1}^{\prime}}, g_{\mu^{\prime}} p_{g\left(\mu^{\prime}\right) \lambda_{1}^{\prime}}\right)<\mathcal{V}_{1}^{\prime} .
$$

We claim that the admissible pair $\left(\lambda_{1}^{\prime}, \mu^{\prime}\right)$ and the open covering $\mathcal{V}_{1}^{\prime}$ are a lifting index and a lifting mesh for $\left(g, g_{\mu}\right)$, respectively (see diagram (4.9) below). Indeed, suppose that $h: Z \times 0 \rightarrow X_{\lambda_{1}^{\prime}}$ and $H: Z \times I \rightarrow Y_{\mu^{\prime}}$ are maps such that

$$
\left(g_{\mu^{\prime}} p_{g\left(\mu^{\prime}\right) \lambda_{1}^{\prime}} h, H_{0}\right)<\mathcal{V}_{1}^{\prime} .
$$

By (4.5) and (4.6),

$$
\left(f_{\mu^{\prime}} p_{f\left(\mu^{\prime}\right) \lambda_{1}^{\prime}} h, H_{0}\right)<\mathcal{V}^{\prime} .
$$

So there is a map $\tilde{H}: Z \times I \rightarrow X_{\lambda_{1}}$ such that

$$
\begin{array}{r}
\left(f_{\mu} p_{f(\mu) \lambda_{1}} \tilde{H}, q_{\mu \mu^{\prime}} H\right)<\mathcal{V}_{1}, \\
\left(p_{\lambda_{1} \lambda_{1}^{\prime}} h, \tilde{H}_{0}\right)<p_{\lambda \lambda_{1}}^{-1} \mathcal{U} .
\end{array}
$$


By (4.4), (4.7), and (4.8),

$$
\begin{aligned}
& \left(g_{\mu} p_{g(\mu) \lambda_{1}} \tilde{H}, q_{\mu \mu^{\prime}} H\right)<\text { st } \mathcal{V}_{1}<\mathcal{V}, \\
& \left(p_{\lambda \lambda_{1}^{\prime}} h, p_{\lambda \lambda_{1}} \tilde{H}_{0}\right)<\mathcal{U},
\end{aligned}
$$

as required.

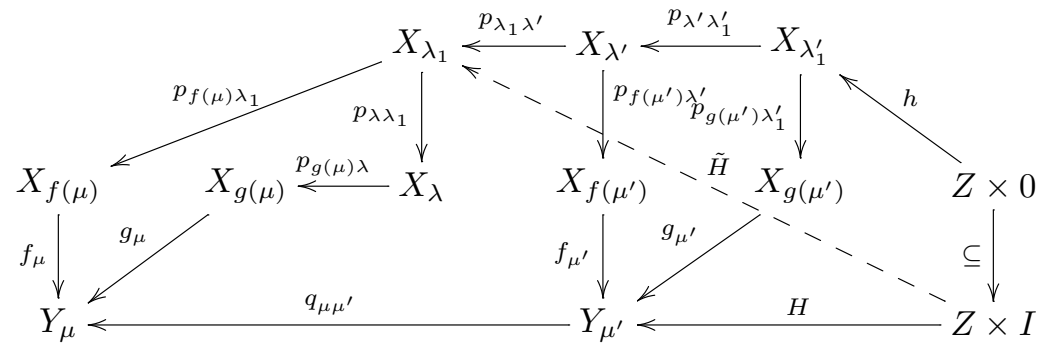

A morphism $\boldsymbol{f}: \boldsymbol{X} \rightarrow \boldsymbol{Y}$ in Apro- ANR is said to have the approximate homotopy lifting property (AHLP) with respect to a space $Z$ provided $\boldsymbol{f}$ is represented by a system map $\left(f, f_{\mu}\right)$ which has the AHLP with respect to $Z$. This is equivalent to saying that every system map $\left(f, f_{\mu}\right)$ representing $\boldsymbol{f}$ has the AHLP with respect to $Z$.

For the rest of this section, let $\boldsymbol{X}$ and $\boldsymbol{Y}$ have the same index set, and write $\boldsymbol{X}=\left(X_{\lambda}, p_{\lambda \lambda^{\prime}}, \Lambda\right)$ and $\boldsymbol{Y}=\left(Y_{\lambda}, q_{\lambda \lambda^{\prime}}, \Lambda\right)$.

For level maps, we have the following characterization of the AHLP [3, Lemma 4.3]:

Proposition 4.2. A level map $\left(f_{\lambda}\right): \boldsymbol{X} \rightarrow \boldsymbol{Y}$ has the AHLP with respect to a space $Z$ if and only if it satisfies the following condition:

$(\mathrm{AHLP})_{L}$ For any $\lambda \in \Lambda$ and for any $\mathcal{U} \in \operatorname{Cov}\left(X_{\lambda}\right)$ and $\mathcal{V} \in \operatorname{Cov}\left(Y_{\lambda}\right)$ there exist $\lambda^{\prime}>\lambda$ and $\mathcal{V}^{\prime} \in \operatorname{Cov}\left(X_{\lambda^{\prime}}\right)$ such that whenever $h: Z \rightarrow X_{\lambda^{\prime}}$ and $H: Z \times I \rightarrow Y_{\lambda^{\prime}}$ are maps with

$$
\left(f_{\lambda^{\prime}} h, H_{0}\right)<\mathcal{V}^{\prime},
$$

then there is a map $\tilde{H}: Z \times I \rightarrow X_{\lambda}$ with

$$
\begin{gathered}
\left(p_{\lambda \lambda^{\prime}} h, \tilde{H}_{0}\right)<\mathcal{U}, \\
\left(f_{\lambda} \tilde{H}, q_{\lambda \lambda^{\prime}} H\right)<\mathcal{V} .
\end{gathered}
$$

If $\boldsymbol{X}$ and $\boldsymbol{Y}$ are ANR-systems, we have the following characterization of property $(\mathrm{AHLP})_{L}$ :

Proposition 4.3. Let $\left(f_{\lambda}\right): \boldsymbol{X} \rightarrow \boldsymbol{Y}$ be a level morphism. 
(1) If $\boldsymbol{Y}$ is an ANR-system, and if the level map $\left(f_{\lambda}\right)$ has the formally weaker lifting property $(W A H L P)_{L}$ with respect to a paracompact space $Z$ which is obtained from $(A H L P)_{L}$ by replacing (4.10) by the equality $f_{\lambda^{\prime}} h=H_{0}$, then it has property (AHLP) with respect to $Z$.

(2) If $\boldsymbol{X}$ is an ANR-system, and if the level map $\left(f_{\lambda}\right)$ has property (AHLP) with respect to a paracompact space $Z$, then it has the formally stronger lifting property $(S A H L P)_{L}$ with respect to $Z$ which is obtained from $(A H L P)_{L}$ by replacing (4.11) by the equality

$$
p_{\lambda \lambda^{\prime}} h=\tilde{H}_{0} .
$$

Proof. (1) is proven by the modification of the proof of $[14$, Proposition $4.3]$, and (2) is proven in [4, Theorem 3.2].

A system map $\left(f, f_{\mu}\right): \boldsymbol{X} \rightarrow \boldsymbol{Y}$ has the homotopy lifting property (HLP) with respect to a space $Z$ provided it satisfies the property (HLP) which is obtained from property (AHLP) by replacing (4.1), (4.2), (4.3) by the following equalities, respectively:

$$
\begin{aligned}
& f_{\mu^{\prime}} p_{f\left(\mu^{\prime}\right) \lambda^{\prime}} h=H_{0}, \\
& p_{\lambda \lambda^{\prime}} h=\tilde{H}_{0}, \\
& f_{\mu} p_{f(\mu) \lambda} \tilde{H}=q_{\mu \mu^{\prime}} H .
\end{aligned}
$$

We have the following characterization of property (HLP) (see [3, Lemma 4.3]):

Proposition 4.4. A level map $\left(f_{\lambda}\right): \boldsymbol{X} \rightarrow \boldsymbol{Y}$ has the HLP with respect to a space $Z$ if and only if it satisfies the following condition:

$(\mathrm{HLP})_{L}$ For any $\lambda \in \Lambda$ there exists $\lambda^{\prime} \geq \lambda$ such that whenever $h: Z \times 0 \rightarrow X_{\lambda^{\prime}}$ and $H: Z \times I \rightarrow Y_{\lambda^{\prime}}$ are maps with

$$
f_{\lambda^{\prime}} h=H_{0},
$$

there exists a map $\tilde{H}: Z \times I \rightarrow X_{\lambda}$ such that

$$
\begin{aligned}
& p_{\lambda \lambda^{\prime}} h=\tilde{H}_{0}, \\
& f_{\lambda} \tilde{H}=q_{\lambda \lambda^{\prime}} H .
\end{aligned}
$$

Note here that property (HLP) is not invariant in pro- ANR (see $[8$, Remark 4]) and hence is not invariant in Apro- ANR. However, Propositions 4.2, 4.3 (1) and 4.4 immediately imply

Corollary 4.5. If a level map $\left(f_{\lambda}\right): \boldsymbol{X} \rightarrow \boldsymbol{Y}$ between ANR-systems has the HLP with respect to a paracompact space $Z$, then it has the AHLP with respect to $Z$.

Finally in this section, we recall the definition of fibration category in the sense of Baues [2]. A fibration category is a category $\mathcal{F}$ with the structure $(\mathcal{F}$, fib, we) which satisfies axioms (F1), (F2), (F3), (F4) below. Here fib 
and we are classes of morphisms, called fibrations and weak equivalences, respectively.

(F1) Composition axiom. The isomorphisms in $\mathcal{F}$ are weak equivalences and fibrations. For any morphisms $f: X \rightarrow Y$ and $g: Y \rightarrow Z$, if any two of $f, g$, and $g \circ f$ are weak equivalences, so is the third. The composite of fibrations is a fibration.

(F2) Pull-back axiom. For any 2 -sink $B \stackrel{g}{\longrightarrow} Y \stackrel{f}{\longleftarrow} X$ in $\mathcal{F}$ with $f$ being a fibration, there is a pull-back diagram in $\mathcal{F}$

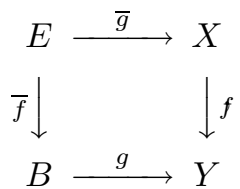

where $\bar{f}$ is a fibration. Moreover, if $f$ ( $g$, respectively) is a weak equivalence, so is $\bar{f}(\bar{g}$, respectively).

(F3) Factorization axiom. Each morphism $f: X \rightarrow Y$ admits a factorization

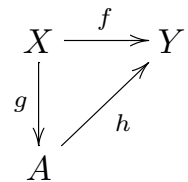

where $g$ is a weak equivalence and $h$ is a fibration.

(F4) Axiom on cofibrant models. Each object $X$ in $\mathcal{F}$ admits a trivial fibration (i.e., a morphism which is both a fibration and a weak equivalence) $R X \rightarrow X$ where $R X$ is a cofibrant in $\mathcal{F}$. An object $R$ is a cofibrant if each trivial fibration $f: Q \rightarrow R$ admits a morphism $s: R \rightarrow Q$ such that $f \circ s=1_{R}$.

The category Top has structures of a fibration category if fibration $=$ Hurewicz fibration, and weak equivalence = homotopy equivalence.

\section{Compositions AXIOM}

THEOREM 5.1. A system map $\left(f, f_{\mu}\right): \boldsymbol{X} \rightarrow \boldsymbol{Y}$ which induces an isomorphism $\boldsymbol{f}: \boldsymbol{X} \rightarrow \boldsymbol{Y}$ in Apro-ANR has the AHLP with respect to any space. Hence every isomorphism $\boldsymbol{f}: \boldsymbol{X} \rightarrow \boldsymbol{Y}$ in Apro-ANR has the AHLP with respect to any space.

Proof. Let $\boldsymbol{g}: \boldsymbol{Y} \rightarrow \boldsymbol{X}$ be the inverse of $\boldsymbol{f}$, and let $\boldsymbol{g}$ be represented by a system map $\left(g, g_{\lambda}\right)$. To show that $\left(f, f_{\mu}\right)$ has the AHLP with respect to any space $Z$, let $(\lambda, \mu) \in \Lambda \times M$ be any admissible pair, and let $\mathcal{U} \in$ $\operatorname{Cov}\left(X_{\lambda}\right)$ and $\mathcal{V} \in \operatorname{Cov}\left(Y_{\mu}\right)$. Choose $\mathcal{U}^{\prime} \in \operatorname{Cov}\left(X_{\lambda}\right)$ such that st $\mathcal{U}^{\prime}<\mathcal{U}$. By $\left(g, g_{\lambda}\right) \circ\left(f, f_{\mu}\right) \sim_{a}\left(1_{\Lambda}, 1_{X_{\lambda}}\right)$, there is $\lambda_{1}>\lambda, f g(\lambda)$ such that

$$
\left(p_{\lambda \lambda_{1}}, g_{\lambda} f_{g(\lambda)} p_{f g(\lambda) \lambda_{1}}\right)<\mathcal{U}^{\prime} .
$$


By $\left(f, f_{\mu}\right) \circ\left(g, g_{\lambda}\right) \sim_{a}\left(1_{M}, 1_{Y_{\mu}}\right)$ and the fact that $\left(g, g_{\lambda}\right)$ is a system map, there is $\mu^{\prime}>\mu, g f(\mu), g(\lambda)$ such that

$$
\begin{gathered}
\left(q_{\mu \mu^{\prime}}, f_{\mu} g_{f(\mu)} q_{g f(\mu) \mu^{\prime}}\right)<\mathcal{V}, \\
p_{f(\mu) \lambda} g_{\lambda} q_{g(\lambda) \mu^{\prime}}=g_{f(\mu)} q_{g f(\mu) \mu^{\prime}} .
\end{gathered}
$$

Let

$$
\mathcal{V}^{\prime}=q_{\mu \mu^{\prime}}^{-1} \mathcal{V} \wedge q_{g(\lambda) \mu^{\prime}}^{-1} g_{\lambda}^{-1} \mathcal{U}^{\prime}
$$

By the fact that $\left(f, f_{\mu}\right)$ is a system map, there is $\lambda^{\prime}>\lambda_{1}, f\left(\mu^{\prime}\right)$ such that

$$
f_{g(\lambda)} p_{f g(\lambda) \lambda^{\prime}}=q_{g(\lambda) \mu^{\prime}} f_{\mu^{\prime}} p_{f\left(\mu^{\prime}\right) \lambda^{\prime}},
$$

Then $\left(\lambda^{\prime}, \mu^{\prime}\right)$ is a lifting index, and $\mathcal{V}^{\prime}$ is a lifting mesh (see diagram (5.7) below). Indeed, suppose that $h: Z \times 0 \rightarrow X_{\lambda^{\prime}}$ and $H: Z \times I \rightarrow Y_{\mu^{\prime}}$ are maps such that

$$
\left(f_{\mu^{\prime}} p_{f\left(\mu^{\prime}\right) \lambda^{\prime}} h, H_{0}\right)<\mathcal{V}^{\prime}
$$

By (5.1), (5.4), (5.5) and (5.6),

$$
\left(g_{\lambda} q_{g(\lambda) \mu^{\prime}} H_{0}, p_{\lambda \lambda^{\prime}} h\right)<\mathcal{U}
$$

By (5.2) and (5.3),

$$
\left(q_{\mu \mu^{\prime}} H, f_{\mu} p_{f(\mu) \lambda} g_{\lambda} q_{g(\lambda) \mu^{\prime}} H\right)<\mathcal{V} .
$$

Thus the map $\tilde{H}: Z \times I \rightarrow X_{\lambda}$ defined by $\tilde{H}=g_{\lambda} q_{g(\lambda) \mu^{\prime}} H$ is the desired homotopy.

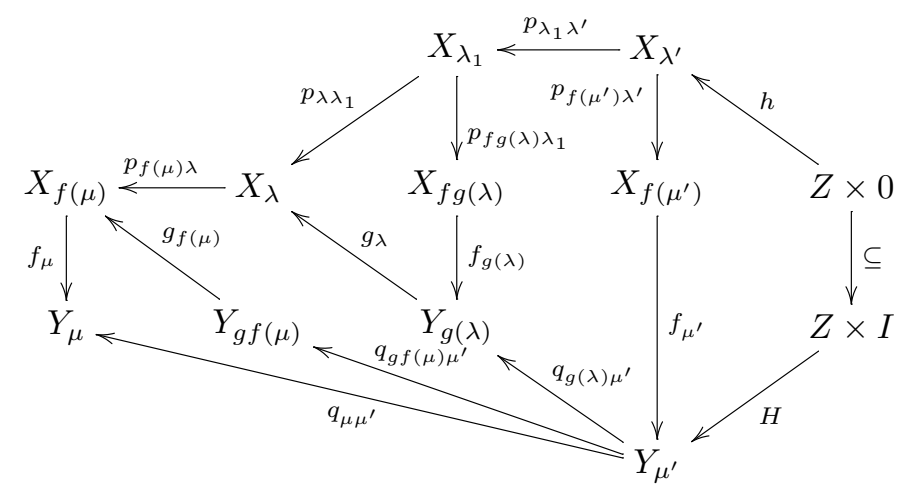

Proposition 5.2. Let $\boldsymbol{f}: \boldsymbol{X} \rightarrow \boldsymbol{Y}$ and $\boldsymbol{g}: \boldsymbol{Y} \rightarrow \boldsymbol{Z}$ be morphisms in Apro- ANR. If $\boldsymbol{f}$ and $\boldsymbol{g}$ have the AHLP with respect to a space $Z$, so does $g \circ f$. 
Proof. It is proven in [3, Proposition 5.4] that if $\left(f, f_{\mu}\right): \boldsymbol{X} \rightarrow \boldsymbol{Y}$ and $\left(g, g_{\nu}\right): \boldsymbol{Y} \rightarrow \boldsymbol{Z}$ represent the morphisms $\boldsymbol{f}$ and $\boldsymbol{g}$, respectively, and if $\left(f, g_{\mu}\right)$ and $\left(g, g_{\nu}\right)$ have the AHLP, so does the composition $\left(g, g_{\nu}\right) \circ\left(f, f_{\mu}\right)$. This immediately implies the theorem.

For any ANR-system $\boldsymbol{X}=\left(X_{\lambda}, p_{\lambda \lambda^{\prime}}, \Lambda\right)$, let $[\boldsymbol{X}]$ denote the induced system $\left(X_{\lambda},\left[p_{\lambda \lambda^{\prime}}\right], \Lambda\right)$ in the homotopy category H(ANR) of ANR. Here for any map $f: X \rightarrow Y$, let $[f]$ denote the homotopy class of the map $f$. Any morphism $\boldsymbol{f}: \boldsymbol{X} \rightarrow \boldsymbol{Y}$ in Apro-ANR induces a morphism $[\boldsymbol{f}]:[\boldsymbol{X}] \rightarrow[\boldsymbol{Y}]$ in pro- H(ANR). Indeed, let $\boldsymbol{f}$ be represented by a system map $\left(f, f_{\mu}\right)$ : $\boldsymbol{X} \rightarrow \boldsymbol{Y}$. Then $\left(f, f_{\mu}\right)$ induces a system map $\left(f,\left[f_{\mu}\right]\right):[\boldsymbol{X}] \rightarrow[\boldsymbol{Y}]$, and let $[\boldsymbol{f}]$ be the morphism represented by $\left(\left[f_{\mu}\right], f\right)$. This is well-defined since if $\left(f, f_{\mu}\right) \sim_{a}\left(g, g_{\mu}\right)$, then for any $\mu \in M$ and for any $\mathcal{V} \in \operatorname{Cov}\left(Y_{\mu}\right)$ so that any $\mathcal{V}$-near maps into $Y_{\mu}$ are homotopic, there is $\lambda>f(\mu), g(\mu)$ such that $\left(f_{\mu} p_{f(\mu) \lambda}, g_{\mu} p_{g(\mu) \lambda}\right)<\mathcal{V}$, which means $f_{\mu} p_{f(\mu) \lambda} \simeq g_{\mu} p_{g(\mu) \lambda}$.

Then we immediately have

Proposition 5.3. Consider a sequence $\boldsymbol{X} \stackrel{\boldsymbol{f}}{\longrightarrow} \boldsymbol{Y} \stackrel{\boldsymbol{g}}{\longrightarrow} \boldsymbol{Z}$ in Apro- ANR. If any two of $\boldsymbol{f}, \boldsymbol{g}, \boldsymbol{g} \boldsymbol{f}$ induce isomorphisms in pro- H(ANR), so does the third.

By Theorem 5.1 and Propositions 5.2 and 5.3 we have

COROLlary 5.4. The category Apro- ANR together with fibration = morphism with the AHLP with respect to any spaces, weak equivalence $=$ morphism inducing an isomorphism in pro- H(ANR) satisfies composition axiom.

\section{FACTORIZATION AXIOM}

ThEOREM 6.1. Every morphism $\boldsymbol{f}: \boldsymbol{X} \rightarrow \boldsymbol{Y}$ in Apro-ANR admits a commutative diagram in Apro- ANR

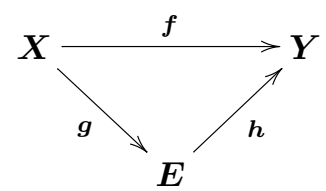

where $\boldsymbol{g}$ is a morphism inducing an isomorphism in pro- $\mathrm{H}(\mathrm{ANR})$ and $\boldsymbol{h}$ is a morphism with the AHLP with respect to any space.

Proof. Recall the construction of the system $\boldsymbol{E}$ from the proof of [14, Theorem 6.1]. In view of Propositions 2.3 and 2.4, we can assume that $\boldsymbol{f}$ is represented by a level map $\left(f_{\lambda}\right): \boldsymbol{X} \rightarrow \boldsymbol{Y}$ and that $\boldsymbol{X}=\left(X_{\lambda}, p_{\lambda \lambda^{\prime}}, \Lambda\right)$ and $\boldsymbol{Y}=\left(Y_{\lambda}, q_{\lambda \lambda^{\prime}}, \Lambda\right)$ are indexed by a cofinite directed set $\Lambda$. We define $\mathcal{V}_{\lambda} \in \operatorname{Cov}\left(Y_{\lambda}\right)$ by induction on the number of predecessors of the elements of $\Lambda$. If $\lambda \in \Lambda$ has no predecessors, then we let $\mathcal{V}_{\lambda}$ be any open covering of $Y_{\lambda}$. If we have defined open coverings $\mathcal{V}_{\lambda}$ for all $\lambda \in \Lambda$ that have at most $n-1$ predecessors, and if $\lambda \in \Lambda$ has $n$ predecessors, then we define an open covering 
$\mathcal{V}_{\lambda}$ of $Y_{\lambda}$ as follows: If $\lambda_{i}, i=1, \ldots, n$, are the predecessors of $\lambda$, then for each $i$, we take an open covering $\mathcal{U}_{i} \in \operatorname{Cov}\left(Y_{\lambda_{i}}\right)$ such that

$$
\mathcal{U}_{i}<\mathcal{V}_{\lambda_{i}} \text {, and }
$$

$$
\text { any two } \mathcal{U}_{i} \text {-near maps into } Y_{\lambda_{i}} \text { are } \mathcal{V}_{\lambda_{i}} \text {-homotopic. }
$$

We then take an open covering $\mathcal{V}_{\lambda} \in \operatorname{Cov}\left(Y_{\lambda}\right)$ such that

$$
\mathcal{V}_{\lambda}<\bigwedge_{i=1}^{n} q_{\lambda_{i} \lambda}^{-1} \mathcal{U}_{i}
$$

For each $\lambda \in \Lambda$, let

$$
E_{\lambda}=\left\{(x, \omega) \in X_{\lambda} \times Y_{\lambda}^{I}:\left(f_{\lambda}(x), \omega(0)\right)<\mathcal{V}_{\lambda}\right\}
$$

Then $E_{\lambda}$ are ANR's since it is an open subset of the ANR $X_{\lambda} \times Y_{\lambda}^{I}$. Since $\mathcal{V}_{\lambda^{\prime}}<q_{\lambda \lambda^{\prime}}^{-1} \mathcal{V}_{\lambda}$ for $\lambda<\lambda^{\prime}$ by (6.2) and (6.4), then there is a well-defined map

$$
r_{\lambda \lambda^{\prime}}: E_{\lambda^{\prime}} \rightarrow E_{\lambda}: r_{\lambda \lambda^{\prime}}(x, \omega)=\left(p_{\lambda \lambda^{\prime}}(x), q_{\lambda \lambda^{\prime}} \omega\right) \text { for } \lambda<\lambda^{\prime} .
$$

For each $\lambda \in \Lambda$, we define maps

$$
g_{\lambda}: X_{\lambda} \rightarrow E_{\lambda}: g_{\lambda}(x)=\left(x, e_{f_{\lambda}(x)}\right) \text { for } x \in X_{\lambda},
$$

and

$$
h_{\lambda}: E_{\lambda} \rightarrow Y_{\lambda}: h_{\lambda}(x, \omega)=\omega(1) \text { for }(x, \omega) \in E_{\lambda} .
$$

Here for any space $W$ and for any $w_{0} \in W$, let $e_{w_{0}} \in W^{I}$ denote the constant path defined by $e_{w_{0}}(t)=w_{0}$ for $t \in I$. Then there is a commutative diagram:

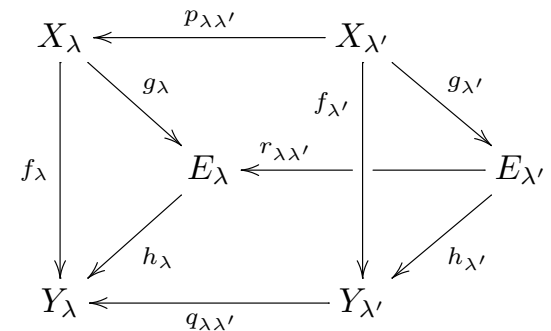

Then we have an ANR-sytem $\boldsymbol{E}=\left(E_{\lambda}, r_{\lambda \lambda^{\prime}}, \Lambda\right)$ and level maps $\left(g_{\lambda}\right): \boldsymbol{X} \rightarrow \boldsymbol{E}$ and $\left(h_{\lambda}\right): \boldsymbol{E} \rightarrow \boldsymbol{Y}$ which induce the commutative diagram (6.1).

The system map $\left(g_{\lambda}\right): \boldsymbol{X} \rightarrow \boldsymbol{E}$ has the AHLP with respect to any spaces since each $g_{\lambda}$ has the homotopy lifting property with respect to any spaces (see [11, Theorem 6.5.10]). Moreover, using (6.3) we can show that the system map $\left(h_{\lambda}\right): \boldsymbol{E} \rightarrow \boldsymbol{Y}$ induces an isomorphism in pro- H(ANR) (see [14, Theorem 6.1]). 


\section{Pull-Backs}

Lemma 7.1. Every 2-sink $\boldsymbol{Y} \stackrel{g}{\longrightarrow} \boldsymbol{Z} \stackrel{f}{\longleftarrow} \boldsymbol{X}$ in Apro- $\mathcal{C}$ admits commutative approximate systems $\mathfrak{X}=\left(X_{\xi}^{\prime}, \mathcal{U}_{\xi}, p_{\xi \xi^{\prime}}^{\prime}, \Xi\right), \mathfrak{Y}=\left(Y_{\xi}^{\prime}, \mathcal{V}_{\xi}, q_{\xi \xi^{\prime}}^{\prime}, \Xi\right)$, and $\mathfrak{Z}=\left(Z_{\xi}^{\prime}, \mathcal{W}_{\xi}, r_{\xi \xi^{\prime}}^{\prime}, \Xi\right)$ and a commutative diagram in Apro- $\mathcal{C}$

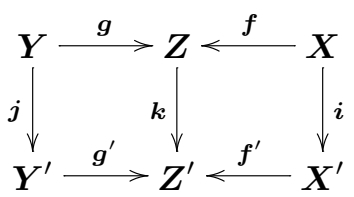

where $\boldsymbol{X}^{\prime}=\left(X_{\xi}^{\prime}, p_{\xi \xi^{\prime}}^{\prime}, \Xi\right), \boldsymbol{Y}^{\prime}=\left(Y_{\xi}^{\prime}, q_{\xi \xi^{\prime}}^{\prime}, \Xi\right)$, and $\boldsymbol{Z}^{\prime}=\left(Z_{\xi}^{\prime}, r_{\xi \xi^{\prime}}^{\prime}, \Xi\right)$ are the systems induced by $\mathfrak{X}, \mathfrak{Y}$, and $\mathfrak{Z}$, respectively, and they satisfy the following conditions:

(1) the index set $\Xi$ is a cofinite directed set,

(2) the terms and bonding maps of $\boldsymbol{X}^{\prime}, \boldsymbol{Y}^{\prime}$, and $\boldsymbol{Z}^{\prime}$ are those of $\boldsymbol{X}, \boldsymbol{Y}$, and $\boldsymbol{Z}$, respectively,

(3) $\mathcal{W}_{\xi^{\prime}}<r_{\xi \xi^{\prime}}^{\prime-1} \mathcal{W}_{\xi}$ for $\xi<\xi^{\prime}$

(4) $\boldsymbol{i}, \boldsymbol{j}$, and $\boldsymbol{k}$ are isomorphisms, and

(5) $\boldsymbol{f}^{\prime}$ and $\boldsymbol{g}^{\prime}$ are represented by level morphisms.

Moreover, if $\boldsymbol{Z}$ is a system in $\mathrm{CM}$,

(6) we can choose the open coverings $\mathcal{W}_{\xi}$ as the open coverings by $\varepsilon_{\xi}$ balls for some $\varepsilon_{\xi}>0$ such that $\mathrm{d}\left(z, z^{\prime}\right) \leq \varepsilon_{\xi^{\prime}}, z, z^{\prime} \in Z_{\xi^{\prime}}$, implies $\mathrm{d}\left(r_{\xi \xi^{\prime}}^{\prime}(z), r_{\xi \xi^{\prime}}^{\prime}\left(z^{\prime}\right)\right)<\varepsilon_{\xi}$.

Proof. Let $\boldsymbol{X}=\left(X_{\lambda}, p_{\lambda \lambda^{\prime}}, \Lambda\right), \boldsymbol{Y}=\left(Y_{\mu}, q_{\mu \mu^{\prime}}, M\right)$, and $\boldsymbol{Z}=\left(Z_{\nu}, r_{\nu \nu^{\prime}}, N\right)$, and let $\boldsymbol{f}$ and $\boldsymbol{g}$ be represented by $\left(f, f_{\nu}\right)$ and $\left(g, g_{\nu}\right)$, respectively. By $[9$, Lemma 2 , Ch. I, $\S 1.2]$ and by the functor $F:$ pro- $\mathcal{C} \rightarrow$ Apro- $\mathcal{C}$, we can assume that $f$ and $g$ are increasing functions. By Proposition 3.2 there exist commutative approximate systems $\mathfrak{X}=\left(\bar{X}_{\alpha}, \mathcal{U}_{\alpha}, \bar{p}_{\alpha \alpha^{\prime}}, \bar{\Lambda}\right), \mathfrak{Y}=\left(\bar{Y}_{\beta}, \mathcal{V}_{\beta}, q_{\beta \beta^{\prime}}, \bar{M}\right)$, and $\mathfrak{Z}=\left(\bar{Z}_{\gamma}, \mathcal{W}_{\gamma}, \bar{r}_{\gamma \gamma^{\prime}}, \bar{N}\right)$ and system maps $\left(\bar{f}, \bar{f}_{\gamma}\right): \overline{\boldsymbol{X}} \rightarrow \overline{\boldsymbol{Z}}$ and $\left(\bar{g}, \bar{g}_{\gamma}\right): \overline{\boldsymbol{Y}} \rightarrow \overline{\boldsymbol{Z}}$ with the properties in Proposition 3.2, where $\overline{\boldsymbol{X}}, \overline{\boldsymbol{Y}}$, and $\overline{\boldsymbol{Z}}$ are the systems induced by $\mathfrak{X}, \mathfrak{Y}$, and $\mathfrak{Z}$, respectively. Then we have the following commutative diagram:

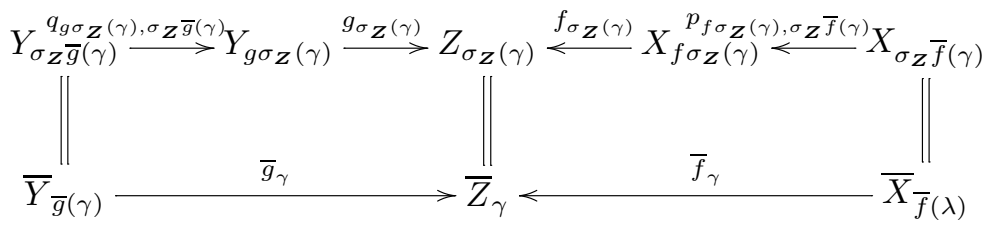


So we have the commutative diagram in Apro- $\mathcal{C}$ :

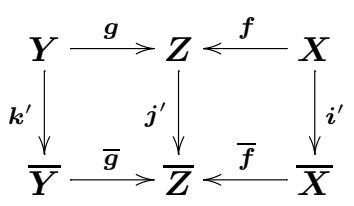

where $\overline{\boldsymbol{f}}$ and $\overline{\boldsymbol{g}}$ are morphisms in Apro- $\mathcal{C}$ represented by $\left(\bar{f}, \bar{f}_{\gamma}\right)$ and $\left(\bar{g}, \bar{g}_{\gamma}\right)$, respectively, and $\boldsymbol{i}^{\prime}, \boldsymbol{j}^{\prime}$, and $\boldsymbol{k}^{\prime}$ are isomorphisms represented by $\left(\sigma_{\boldsymbol{X}}, 1_{\sigma_{\boldsymbol{X}}(\alpha)}\right)$, $\left(\sigma_{\boldsymbol{Y}}, 1_{\sigma_{\boldsymbol{Y}}(\beta)}\right)$, and $\left(\sigma_{\boldsymbol{Z}}, 1_{\sigma_{\boldsymbol{Z}}(\gamma)}\right)$, respectively.

Let $\Xi$ be the set of triples $(\alpha, \beta, \gamma) \in \bar{\Lambda} \times \bar{M} \times \bar{N}$ such that $\alpha>\bar{f}(\gamma)$ and $\beta \geq \bar{g}(\gamma)$, and let $\Xi$ be ordered by $(\alpha, \beta, \gamma) \leq\left(\alpha^{\prime}, \beta^{\prime}, \gamma^{\prime}\right)$ if and only if $\alpha \leq \alpha^{\prime}, \beta \leq \beta^{\prime}$, and $\gamma \leq \gamma^{\prime}$. For each $\xi=(\alpha, \beta, \gamma) \in \Xi$, let $X_{\xi}^{\prime}=\bar{X}_{\alpha}$, $Y_{\xi}^{\prime}=\bar{Y}_{\beta}, Z_{\xi}^{\prime}=\bar{Z}_{\gamma}, \mathcal{U}_{\xi}^{\prime}=\mathcal{U}_{\alpha}, \mathcal{V}_{\xi}^{\prime}=\mathcal{V}_{\beta}, \mathcal{W}_{\xi}^{\prime}=\mathcal{W}_{\gamma}$, and define maps $p_{\xi \xi^{\prime}}^{\prime}=$ $\bar{p}_{\alpha \alpha^{\prime}}: X_{\xi^{\prime}}^{\prime} \rightarrow X_{\xi}^{\prime}, q_{\xi \xi^{\prime}}^{\prime}=\bar{q}_{\beta \beta^{\prime}}: Y_{\xi^{\prime}}^{\prime} \rightarrow Y_{\xi}^{\prime}, r_{\xi \xi^{\prime}}^{\prime}=\bar{r}_{\gamma \gamma^{\prime}}: Z_{\xi^{\prime}}^{\prime} \rightarrow Z_{\xi}^{\prime}$. Then $\mathfrak{X}^{\prime}=\left(X_{\xi}^{\prime}, \mathcal{U}_{\xi}^{\prime}, p_{\xi \xi^{\prime}}^{\prime}, \Xi\right), \mathfrak{Y}^{\prime}=\left(Y_{\xi}^{\prime}, \mathcal{V}_{\xi}^{\prime}, q_{\xi \xi^{\prime}}^{\prime}, \Xi\right)$, and $\mathfrak{Z}^{\prime}=\left(Z_{\xi}^{\prime}, \mathcal{W}_{\xi}^{\prime}, r_{\xi \xi^{\prime}}^{\prime}, \Xi\right)$ form commutative approximate systems. For each $\xi=(\alpha, \beta, \gamma) \in \Xi$, we define maps $f_{\xi}^{\prime}: X_{\xi}^{\prime} \rightarrow Z_{\xi}^{\prime}: f_{\xi}^{\prime}=\bar{f}_{\gamma} \bar{p}_{\bar{f}(\gamma) \alpha}$ and $g_{\xi}^{\prime}: Y_{\xi}^{\prime} \rightarrow Z_{\xi}^{\prime}: g_{\xi}^{\prime}=\bar{g}_{\gamma} \bar{q}_{\bar{g}(\gamma) \beta}$. For $\xi \leq \xi^{\prime}=\left(\alpha^{\prime}, \beta^{\prime}, \gamma^{\prime}\right)$, we have the commutative diagram:

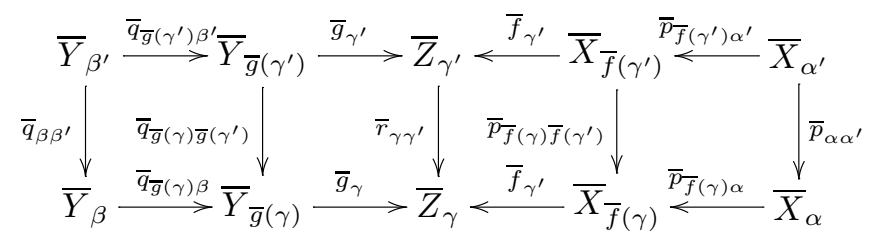

So we have level maps $\left(f_{\xi}^{\prime}\right): \boldsymbol{X}^{\prime} \rightarrow \boldsymbol{Z}^{\prime}$ and $\left(g_{\xi}^{\prime}\right): \boldsymbol{Y}^{\prime} \rightarrow \boldsymbol{Z}^{\prime}$.

We then define system maps $\left(i, i_{\xi}\right): \overline{\boldsymbol{X}} \rightarrow \boldsymbol{X}^{\prime},\left(j, j_{\xi}\right): \overline{\boldsymbol{Y}} \rightarrow \boldsymbol{Y}^{\prime}$, and $\left(k, k_{\xi}\right): \overline{\boldsymbol{Z}} \rightarrow \boldsymbol{Z}^{\prime}$ as follows: First, we define functions $i: \Xi \rightarrow \bar{\Lambda}, j: \Xi \rightarrow \bar{M}$, $k: \Xi \rightarrow \bar{N}$ by $i(\xi)=\alpha, j(\xi)=\beta, k(\xi)=\gamma$. We define the maps $i_{\xi}=1_{\bar{X}_{\alpha}}$, $j_{\xi}=1_{\bar{Y}_{\beta}}, k_{\xi}=1_{\bar{Z}_{\gamma}}$. For each $\xi=(\alpha, \beta, \gamma) \in \Xi$, there is a commutative diagram:

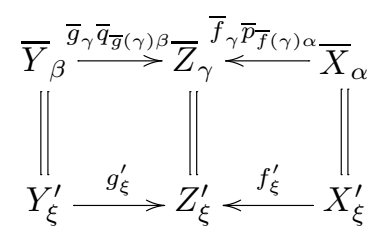


Then we have the following commutative diagram in Apro- $\mathcal{C}$ :

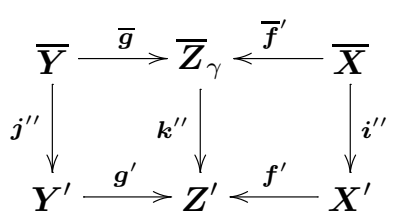

Here $\overline{\boldsymbol{f}}, \overline{\boldsymbol{g}}, \boldsymbol{f}^{\prime}, \boldsymbol{g}^{\prime}, \boldsymbol{i}^{\prime \prime}, \boldsymbol{j}^{\prime \prime}, \boldsymbol{k}^{\prime \prime}$ are the morphisms represented by $\left(\bar{f}, \bar{f}_{\gamma}\right),\left(\bar{g}, \bar{g}_{\gamma}\right)$, $\left(f_{\xi}^{\prime}\right),\left(g_{\xi}^{\prime}\right),\left(i, i_{\xi}\right),\left(j, j_{\xi}\right),\left(k, k_{\xi}\right)$, respectively.

It remains to show that $\boldsymbol{i}^{\prime \prime}, \boldsymbol{j}^{\prime \prime}$, and $\boldsymbol{k}^{\prime \prime}$ are isomorphisms in Apro- $\mathcal{C}$. To show that $\boldsymbol{i}^{\prime \prime}$ is an isomorphism, we define a system map $\left(i^{\prime}, i_{\alpha}^{\prime}\right): \boldsymbol{X}^{\prime} \rightarrow \overline{\boldsymbol{X}}$ such that

$$
\begin{aligned}
& \left(i, i_{\xi}\right) \circ\left(i^{\prime}, i_{\alpha}^{\prime}\right) \sim_{a}\left(1_{\Xi}, 1_{X_{\xi}^{\prime}}\right), \\
& \left(i^{\prime}, i_{\alpha}^{\prime}\right) \circ\left(i, i_{\xi}\right) \sim_{a}\left(1_{\bar{\Lambda}}, 1_{\bar{X}_{\alpha}}\right) .
\end{aligned}
$$

Choose $\gamma_{0} \in \bar{N}$ arbitrarily, and choose $\beta_{0} \in \bar{M}$ such that $\beta_{0} \geq \bar{g}\left(\gamma_{0}\right)$. Choose then an increasing function $\varphi: \bar{\Lambda} \rightarrow \bar{\Lambda}$ such that $\varphi(\alpha) \geq \alpha, \bar{f}\left(\gamma_{0}\right)$. Define the function $i^{\prime}: \bar{\Lambda} \rightarrow \Xi$ by $i^{\prime}(\alpha)=\left(\varphi(\alpha), \beta_{0}, \gamma_{0}\right)$, and define the map $i_{\alpha}^{\prime}: X_{i^{\prime}(\alpha)}^{\prime} \rightarrow$ $\bar{X}_{\alpha}$ by $i_{\alpha}^{\prime}=\bar{p}_{\alpha \varphi(\alpha)}$. Then $i_{\xi} i_{i(\xi)}^{\prime}=\bar{p}_{\alpha \varphi(\alpha)}$ and $i_{\alpha}^{\prime} i_{i^{\prime}(\alpha)}=\bar{p}_{\alpha \varphi(\alpha)}$, which means (7.2) and (7.3). Similarly we can show that $\boldsymbol{j}^{\prime \prime}$ and $\boldsymbol{k}^{\prime \prime}$ are isomorphisms.

TheOREM 7.2. Apro-ANR has weak pull-backs.

Proof. Suppose that we have a 2-sink $\boldsymbol{X} \stackrel{f}{\longrightarrow} \boldsymbol{Z} \stackrel{g}{\longleftarrow} \boldsymbol{Y}$ in Apro- ANR. By Lemma 7.1, we can assume that $\boldsymbol{X}, \boldsymbol{Y}$, and $\boldsymbol{Z}$ have the same index set and that $\boldsymbol{f}$ and $\boldsymbol{g}$ are represented by level morphisms $\left(f_{\lambda}\right)$ and $\left(g_{\lambda}\right)$, respectively. Write $\boldsymbol{X}=\left(X_{\lambda}, p_{\lambda \lambda^{\prime}}, \Lambda\right), \boldsymbol{Y}=\left(Y_{\lambda}, q_{\lambda \lambda^{\prime}}, \Lambda\right)$, and $\boldsymbol{Z}=\left(Z_{\lambda}, r_{\lambda \lambda^{\prime}}, \Lambda\right)$. Moreover, we can assume that there exists an associated commutative approximate system $\left(Z_{\lambda}, \mathcal{W}_{\lambda}, q_{\lambda \lambda^{\prime}}, \Lambda\right)$ such that $\mathcal{W}_{\lambda^{\prime}}<r_{\lambda \lambda^{\prime}}^{-1} \mathcal{W}_{\lambda}$ for $\lambda<\lambda^{\prime}$. For each $\lambda \in \Lambda$, let

$$
E_{\lambda}=\left\{(x, y) \in X_{\lambda} \times Y_{\lambda}:\left(f_{\lambda}(x), g_{\lambda}(y)\right)<\mathcal{W}_{\lambda}\right\}
$$

For each $(x, y) \in E_{\lambda^{\prime}}$ and for $\lambda \leq \lambda^{\prime},\left(f_{\lambda^{\prime}}(x), g_{\lambda^{\prime}}(y)\right)<\mathcal{W}_{\lambda^{\prime}}$ implies $\left(f_{\lambda} p_{\lambda \lambda^{\prime}}(x), g_{\lambda} q_{\lambda \lambda^{\prime}}(y)\right)<\mathcal{W}_{\lambda}$, so there is a well-defined map

$$
s_{\lambda \lambda^{\prime}}: E_{\lambda^{\prime}} \rightarrow E_{\lambda}: s_{\lambda \lambda^{\prime}}(x, y)=\left(p_{\lambda \lambda^{\prime}}(x), q_{\lambda \lambda^{\prime}}(y)\right) .
$$

Note that each $E_{\lambda}$ is an ANR since it is an open subset of the product $X_{\lambda} \times Y_{\lambda}$ of two ANR's. Thus we have an ANR-system $\boldsymbol{E}=\left(E_{\lambda}, s_{\lambda \lambda^{\prime}}, \Lambda\right)$ and a square 
in Apro- ANR:

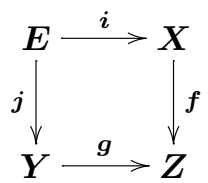

Square (7.5) commutes in Apro- ANR. Indeed, for each $\lambda \in \Lambda$ and for each $\mathcal{W} \in \operatorname{Cov}\left(Z_{\lambda}\right)$ choose $\lambda^{\prime}>\lambda$ such that $\mathcal{W}_{\lambda^{\prime}}<r_{\lambda \lambda^{\prime}}^{-1} \mathcal{W}$. Then

$$
\left(f_{\lambda^{\prime}} i_{\lambda^{\prime}}, g_{\lambda^{\prime}} j_{\lambda^{\prime}}\right)<\mathcal{W}_{\lambda^{\prime}}
$$

This together with the commutativity of diagram (7.6) below implies

$$
\left(f_{\lambda} i_{\lambda} s_{\lambda \lambda^{\prime}}, g_{\lambda} j_{\lambda} s_{\lambda \lambda^{\prime}}\right)<\mathcal{W} \text {. }
$$

This means $\boldsymbol{f} \boldsymbol{i}=\boldsymbol{g} \boldsymbol{j}$ in Apro- ANR.

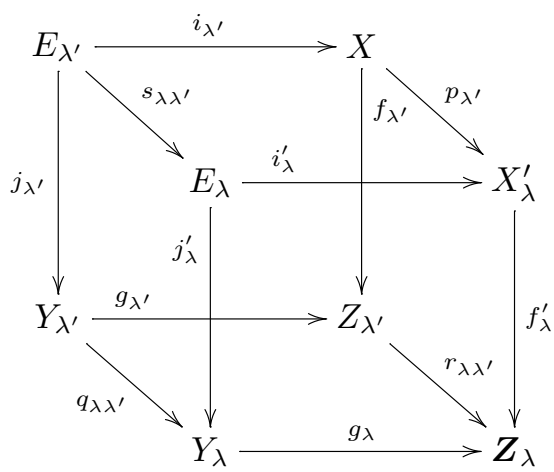

To show that (7.5) is a weak pull-back, consider the following commutative diagram in Apro- ANR:

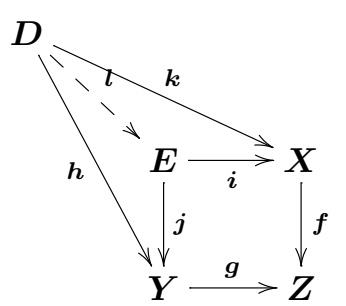

Write $\boldsymbol{D}=\left(D_{\mu}, t_{\mu \mu^{\prime}}, M\right)$, and let $\boldsymbol{k}$ and $\boldsymbol{h}$ be represented by system maps $\left(k, k_{\lambda}\right)$ and $\left(h, h_{\lambda}\right)$, respectively. We must find a morphism $\boldsymbol{l}: \boldsymbol{D} \rightarrow \boldsymbol{E}$ which makes the diagram commute. Since $\boldsymbol{f} \boldsymbol{k}=\boldsymbol{g} \boldsymbol{h}$, for each $\lambda \in \Lambda$ there is $l(\lambda) \in M$ such that $l(\lambda)>k(\lambda), h(\lambda)$ and

$$
\left(f_{\lambda} k_{\lambda} t_{k(\lambda) l(\lambda)}, g_{\lambda} h_{\lambda} t_{h(\lambda) l(\lambda)}\right)<\mathcal{W}_{\lambda} .
$$


So we have a function $l: \Lambda \rightarrow M: \lambda \mapsto l(\lambda)$, and a map $l_{\lambda}: D_{l(\lambda)} \rightarrow E_{\lambda}$ such that $i_{\lambda} l_{\lambda}=k_{\lambda} t_{k(\lambda) l(\lambda)}$ and $j_{\lambda} l_{\lambda}=h_{\lambda} t_{h(\lambda) l(\lambda)}$. Then $\left(l_{\lambda}, l\right): \boldsymbol{D} \rightarrow \boldsymbol{E}$ is a system map. Indeed, by the fact that $\left(k, k_{\lambda}\right)$ and $\left(h, h_{\lambda}\right)$ are system maps, for each $\lambda<\lambda^{\prime}$ there is $\mu>l(\lambda), l\left(\lambda^{\prime}\right)$ such that

$$
\begin{aligned}
& k_{\lambda} t_{k(\lambda) \mu}=p_{\lambda \lambda^{\prime}} k_{\lambda^{\prime}} t_{k\left(\lambda^{\prime}\right) \mu}, \\
& h_{\lambda} t_{h(\lambda) \mu}=q_{\lambda \lambda^{\prime}} h_{\lambda^{\prime}} t_{h\left(\lambda^{\prime}\right) \mu} .
\end{aligned}
$$

This means $l_{\lambda} t_{l(\lambda) \mu}=s_{\lambda \lambda^{\prime}} l_{\lambda^{\prime}} t_{l\left(\lambda^{\prime}\right) \mu}$ as required.

THEOREM 7.3. In the weak pull-back (7.5) if $\boldsymbol{f}$ has the AHLP with respect to any paracompact space $W$, then $\boldsymbol{j}$ has the HLP and hence the AHLP with respect to $W$.

Proof. Assume the setting in the proof of Theorem 7.2. To show that $\left(j_{\lambda}\right)$ has the HLP with respect to $W$, let $\lambda \in \Lambda$ (see diagram (7.9) below). Apply property $(\mathrm{SAHLP})_{L}$ for $\left(f_{\lambda}\right)$ with the index $\lambda$ and $\mathcal{W}_{\lambda} \in \operatorname{Cov}\left(Z_{\lambda}\right)$, and obtain $\lambda^{\prime}>\lambda$ and $\mathcal{W}^{\prime} \in \operatorname{Cov}\left(Z_{\lambda^{\prime}}\right)$ with property (SAHLP) $)_{L}$ (see Proposition $4.3(2))$. Choose $\lambda^{\prime \prime}>\lambda^{\prime}$ such that $\mathcal{W}_{\lambda^{\prime \prime}}<r_{\lambda^{\prime} \lambda^{\prime \prime}}^{-1} \mathcal{W}^{\prime}$. To show that $\lambda^{\prime \prime}$ is a lifting index for $\left(j_{\lambda}\right)$, suppose that $h: W \times 0 \rightarrow E_{\lambda^{\prime \prime}}$ and $H: W \times I \rightarrow Y_{\lambda^{\prime \prime}}$ such that $H_{0}=j_{\lambda^{\prime \prime}} h$. We must find a map $\tilde{H}: W \times I \rightarrow E_{\lambda}$ such that

$$
\tilde{H}_{0}=s_{\lambda \lambda^{\prime \prime}} h,
$$

$$
j_{\lambda} \tilde{H}=q_{\lambda \lambda^{\prime \prime}} H .
$$

Note that

$$
\left(f_{\lambda^{\prime \prime}} i_{\lambda^{\prime \prime}} h, g_{\lambda^{\prime \prime}} H_{0}\right)<\mathcal{W}_{\lambda^{\prime \prime}}
$$

so

$$
\left(r_{\lambda^{\prime} \lambda^{\prime \prime}} f_{\lambda^{\prime \prime}} i_{\lambda^{\prime \prime}} h, r_{\lambda^{\prime} \lambda^{\prime \prime}} g_{\lambda^{\prime \prime}} H_{0}\right)<\mathcal{W}^{\prime}
$$

which means

$$
\left(f_{\lambda^{\prime}} p_{\lambda^{\prime} \lambda^{\prime \prime}} i_{\lambda^{\prime \prime}} h, r_{\lambda^{\prime} \lambda^{\prime \prime}} g_{\lambda^{\prime \prime}} H_{0}\right)<\mathcal{W}^{\prime} .
$$

This together with property $(\mathrm{SAHLP})_{L}$ implies that there is a map $H^{\prime}$ : $W \times I \rightarrow X_{\lambda}$ such that

$$
\begin{aligned}
& \left(f_{\lambda} H^{\prime}, r_{\lambda \lambda^{\prime \prime}} g_{\lambda^{\prime \prime}} H\right)<\mathcal{W}_{\lambda}, \\
& H_{0}^{\prime}=p_{\lambda \lambda^{\prime \prime}} i_{\lambda^{\prime \prime}} h .
\end{aligned}
$$

Thus $H^{\prime}$ and $q_{\lambda \lambda^{\prime \prime}} H$ determine a map $\tilde{H}: W \times I \rightarrow E_{\lambda}$ such that $i_{\lambda} \tilde{H}=H^{\prime}$ and (7.8) holds. Moreover, (7.7) holds since both maps in (7.7) are determined 
by the maps $q_{\lambda \lambda^{\prime \prime}} H_{0}$ and $H_{0}^{\prime}$. This completes the proof of the theorem.

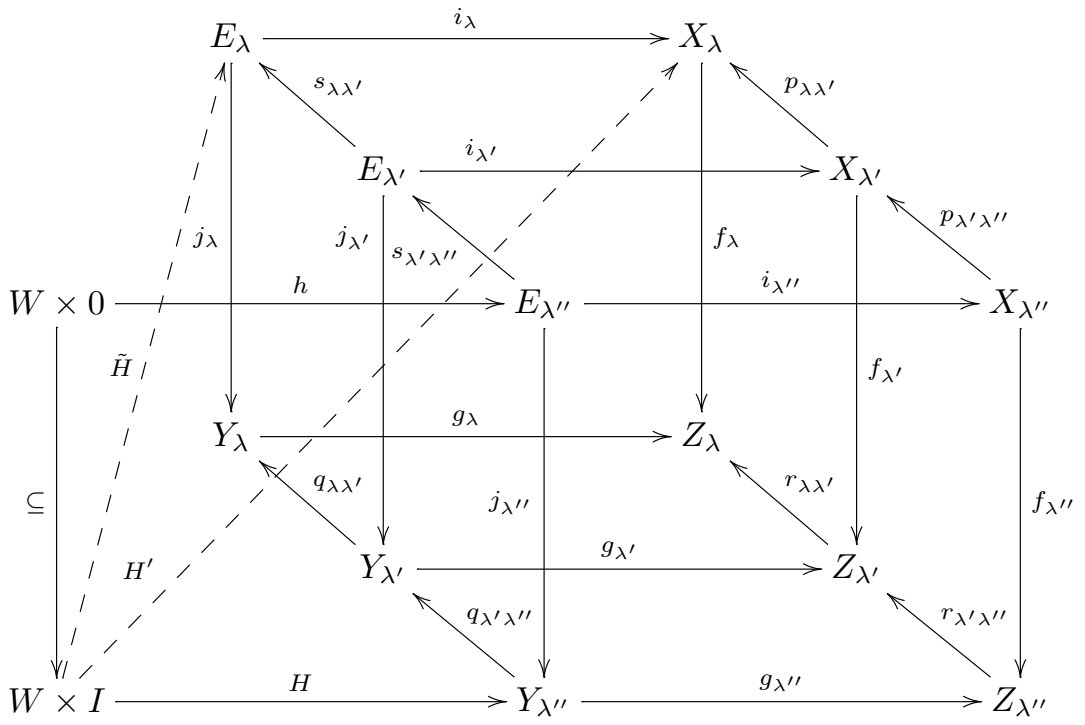

\section{Pull-BACKS in the APPROXimate PRO-CATEGORY OF UNIFORM SPACES}

In this section we discuss the existence of pull-backs in the approximate pro-category of ANRU's.

A uniform space $X$ is an ANRU (uniform absolute neighborhood retract) provided whenever $X$ is embedded in a uniform space $Y$ then there is a uniform retraction of some uniform neighborhood of $X$ in $Y$ onto $X$, equivalently, every uniform map $f: A \rightarrow X$ from a uniform subspace $A$ of a uniform space $Z$ into $X$ extends over some uniform neighborhood of $A$ in $Z$.

Let Unif be the category of uniform spaces and uniform maps, and let ANRU be the full subcategory of Unif whose objects are ANRU's.

For any uniform space $X$, let $\operatorname{Cov}_{U}(X)$ denote the family of all uniform coverings of $X$.

In an analogous way we can show that Propositions 2.1 and 2.2 hold for any subcategory $\mathcal{C}$ of Unif, and hence we can define the approximate procategory Apro- $\mathcal{C}$. Moreover, it is a routine to check that Propositions 2.3, $2.4,2.5,3.1$, and 3.2 hold in the uniform case. Note here that the uniform version of the theory of the approximate resolutions is discussed in [16].

We can also define the AHLP with respect to a uniform space for system maps between ANRU's. Proposition 4.1 holds for the uniform case, and hence the AHLP is well-defined for morphisms in Apro-ANRU. Replacing spaces, maps, coverings by uniform spaces, uniform maps, uniform coverings, 
respectively, we can show that Theorem 5.1 and Proposition 5.2 hold for the morphisms in Apro- ANRU.

In the category Unif we consider a homotopy called the semi-uniform homotopy in the sense of [12]. Two uniform maps $f, g: X \rightarrow Y$ are said to be semi-uniform homotopic provided there exists a uniform map $H: X * I \rightarrow Y$ such that $H_{0}=f$ and $H_{1}=g$. Here $X * I$ denotes the semi-uniform product in the sense of $\left[5\right.$, p. 44]. For any ANRU-system $\boldsymbol{X}=\left(X_{\lambda}, p_{\lambda \lambda^{\prime}}, \Lambda\right)$, we have an induced system $[\boldsymbol{X}]=\left(X_{\lambda},\left[p_{\lambda \lambda^{\prime}}\right], \Lambda\right)$ in the homotopy category H(ANRU). Then, in an analogous way, each morphism $\boldsymbol{f}: \boldsymbol{X} \rightarrow \boldsymbol{Y}$ in Apro- ANRU induces a morphism $[\boldsymbol{f}]:[\boldsymbol{X}] \rightarrow[\boldsymbol{Y}]$ in pro- $\mathrm{H}(\mathrm{ANRU})$. Note here that for any ANRU $Y$, there exists $\mathcal{V} \in \operatorname{Cov}_{U}(Y)$ such that any $\mathcal{V}$-near uniform maps are semi-uniformly homotopic [5, Proposition 16, Chap. V]. Using this notion of homotopy, we have the uniform version of Proposition 5.3.

Moreover, by a completely analogous argument, we see that the uniform version of Theorem 6.1 also holds. Note here that $E_{\lambda}=\left\{(x, \varphi) \in X_{\lambda} \times\right.$ $\left.U\left(I, Y_{\lambda}\right):\left(f_{\lambda}(x), \varphi(0)\right)<\mathcal{V}_{\lambda}\right\}$, where $\times$ denotes the uniform product, is an ANRU, by the facts that if $A$ and $B$ are ANRU's, then the set $U(I, B)$ of all uniform maps $f: I \rightarrow B$ is an ANRU and the uniform product $A \times U(I, B)$ is also an ANRU.

In the uniform case we can improve Theorem 7.2.

THEOREM 8.1. Apro- ANRU has pull-backs.

Proof. For any 2-sink $\boldsymbol{X} \stackrel{\boldsymbol{f}}{\longrightarrow} \boldsymbol{Z} \stackrel{\boldsymbol{g}}{\longleftarrow} \boldsymbol{Y}$ in Apro-ANRU, we define the system $\boldsymbol{E}=\left(E_{\lambda}, s_{\lambda \lambda^{\prime}}, \Lambda\right)$ as in the proof of Theorem 7.2. Here we use the uniform product $\times$ in the definition of $E_{\lambda}$ in (7.4). In the same way we can show that diagram (7.5) is a weak pull-back. So, it remains to show that the morphism $\boldsymbol{l}$ obtained in the proof of Theorem 7.2 is unique. Suppose that we have another morphism $\boldsymbol{l}^{\prime}$ in Apro- ANRU which makes the following diagram commute:

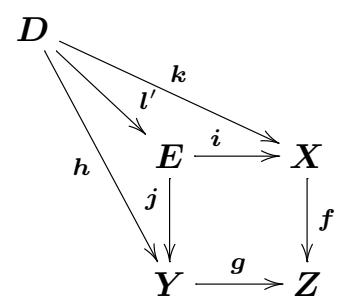

We must show $\boldsymbol{l}=\boldsymbol{l}^{\prime}$. Let $\boldsymbol{l}^{\prime}$ be represented by a system map $\left(l^{\prime}, l_{\lambda}^{\prime}\right)$. To show $\left(l, l_{\lambda}\right) \sim_{a}\left(l^{\prime}, l_{\lambda}^{\prime}\right)$, let $\lambda \in \Lambda$, and let $\mathcal{W} \in \operatorname{Cov}_{U}\left(E_{\lambda}\right)$. Choose $\mathcal{U} \in \operatorname{Cov}_{U}\left(X_{\lambda}\right)$ and $\mathcal{V} \in \operatorname{Cov}_{U}\left(Y_{\lambda}\right)$ such that $\mathcal{U} \times \mathcal{V}<\mathcal{W}$. Then by $\boldsymbol{i l}=\boldsymbol{k}=\boldsymbol{i l}$ and $\boldsymbol{j} \boldsymbol{l}^{\prime}=\boldsymbol{h}=\boldsymbol{j} \boldsymbol{l}$, there exists $\mu>l(\lambda), l^{\prime}(\lambda)$ such that

$$
\begin{aligned}
& \left(i_{\lambda} l_{\lambda} t_{l(\lambda) \mu}, i_{\lambda} l_{\lambda}^{\prime} t_{l^{\prime}(\lambda) \mu}\right)<\mathcal{U}, \\
& \left(j_{\lambda} l_{\lambda} t_{l(\lambda) \mu}, j_{\lambda} l_{\lambda}^{\prime} t_{l^{\prime}(\lambda) \mu}\right)<\mathcal{V},
\end{aligned}
$$


which implies

$$
\left(l_{\lambda}^{\prime} t_{l^{\prime}(\lambda) \mu}, l_{\lambda} t_{l(\lambda) \mu}\right)<\mathcal{U} \times \mathcal{V}<\mathcal{W} .
$$

This shows $\left(l, l_{\lambda}\right) \sim_{a}\left(l^{\prime}, l_{\lambda}^{\prime}\right)$.

Let $\mathrm{ANR}_{c}$ denote the full subcategory of Top whose objects are compact ANR's. Then by the same argument as for Theorem 8.1 we have

Corollary 8.2. Every 2-sink $\boldsymbol{X} \stackrel{\boldsymbol{g}}{\longrightarrow} \boldsymbol{Z} \stackrel{\boldsymbol{f}}{\longleftarrow} \boldsymbol{Y}$ in Apro- $\mathrm{ANR}_{c}$ has a pull-back in Apro- ANR.

\section{EXPANSIONS OF PULL-BACKS IN Apro-ANR}

Let $\boldsymbol{X}=\left(X_{\lambda}, p_{\lambda \lambda^{\prime}}, \Lambda\right)$ and $\boldsymbol{Y}=\left(Y_{\mu}, q_{\mu \mu^{\prime}}, M\right)$ be any ANR $_{c}$-systems with limits $\boldsymbol{p}=\left(p_{\lambda}\right): X \rightarrow \boldsymbol{X}$ and $\boldsymbol{q}=\left(q_{\mu}\right): Y \rightarrow \boldsymbol{Y}$. For any system map $\left(f, f_{\mu}\right): \boldsymbol{X} \rightarrow \boldsymbol{Y}$, a map $\varphi: X \rightarrow Y$ is a limit of $\left(f, f_{\mu}\right)$ provided for any $\mu \in M, q_{\mu} \varphi=f_{\mu} p_{f(\mu)}$. Note that each system map $\left(f, f_{\mu}\right): \boldsymbol{X} \rightarrow \boldsymbol{Y}$ determines a unique limit. It is easy to see that if $\left(f^{\prime}, f_{\mu}^{\prime}\right): \boldsymbol{X} \rightarrow \boldsymbol{Y}$ is a system map such that $\left(f, f_{\mu}\right) \sim_{a}\left(f^{\prime}, f_{\mu}^{\prime}\right)$, and if $\varphi$ is a limit of $\left(f, f_{\mu}\right)$, then it is also the limit of $\left(f^{\prime}, f_{\mu}^{\prime}\right)$. Thus each morphism $f$ in Apro- Top determines a unique limit $\varphi: X \rightarrow Y$, which is denoted by $\lim f$.

TheOrem 9.1. Suppose that we have a pull-back in Apro- ANR

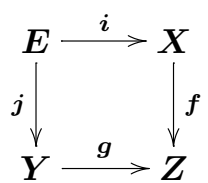

where $\boldsymbol{X}, \boldsymbol{Y}$, and $\boldsymbol{Z}$ are $\mathrm{ANR}_{c}$-systems. Then the limit of the commutative square

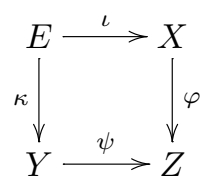

is a pull-back in Top.

Proof. By Lemma 7.1 and Proposition 2.7 we can assume that the systems $\boldsymbol{X}, \boldsymbol{Y}$, and $\boldsymbol{Z}$ have the same index set, say, $\boldsymbol{X}=\left(X_{\lambda}, p_{\lambda \lambda^{\prime}}, \Lambda\right)$, $\boldsymbol{Y}=\left(Y_{\lambda}, q_{\lambda \lambda^{\prime}}, \Lambda\right)$, and $\boldsymbol{Z}=\left(Z_{\lambda}, r_{\lambda \lambda^{\prime}}, \Lambda\right)$, that the morphisms $\boldsymbol{f}, \boldsymbol{g}, \boldsymbol{i}$, and $\boldsymbol{j}$ are represented by level maps $\left(f_{\lambda}\right),\left(g_{\lambda}\right),\left(i_{\lambda}\right)$, and $\left(j_{\lambda}\right)$, and that there is an associated approximate system $\mathfrak{Z}=\left(Z_{\lambda}, \varepsilon_{\lambda}, r_{\lambda \lambda^{\prime}}, \Lambda\right)$ with the following property:

$$
\mathrm{d}\left(z, z^{\prime}\right) \leq \varepsilon_{\lambda^{\prime}}, z, z^{\prime} \in Z_{\lambda^{\prime}} \Longrightarrow \mathrm{d}\left(r_{\lambda \lambda^{\prime}}(z), r_{\lambda \lambda^{\prime}}\left(z^{\prime}\right)\right)<\varepsilon_{\lambda}
$$

Moreover, we can assume that

$$
E_{\lambda}=\left\{(x, y) \in X_{\lambda} \times Y_{\lambda}:\left(f_{\lambda}(x), g_{\lambda}(y)\right)<\varepsilon_{\lambda}\right\}
$$


and that $s_{\lambda \lambda^{\prime}}: E_{\lambda^{\prime}} \rightarrow E_{\lambda}$ is the map defined by $s_{\lambda \lambda^{\prime}}(x, y)=\left(p_{\lambda \lambda^{\prime}}(x), q_{\lambda \lambda^{\prime}}(y)\right)$. Let $\boldsymbol{p}=\left(p_{\lambda}\right): X \rightarrow \boldsymbol{X}, \boldsymbol{q}=\left(q_{\lambda}\right): Y \rightarrow \boldsymbol{Y}, \boldsymbol{r}=\left(r_{\lambda}\right): Z \rightarrow \boldsymbol{Z}$ be limits (hence ANR $_{c}$-resolutions). Let $E=\{(x, y) \in X \times Y: \varphi(x)=\psi(y)\}$, and define the systems map $\boldsymbol{s}=\left(s_{\lambda}\right): E \rightarrow \boldsymbol{E}$ by $s_{\lambda}(x, y)=\left(p_{\lambda}(x), q_{\lambda}(y)\right)$. To show the theorem, it suffices to show that $s=\left(s_{\lambda}\right): E \rightarrow \boldsymbol{E}$ is an ANR-resolution of $E$. To verify this fact, consider the system $\boldsymbol{F}=\left(F_{\lambda}, s_{\lambda \lambda^{\prime}}^{\prime}, \Lambda\right)$ where $F_{\lambda}=$ $\left\{(x, y) \in X_{\lambda} \times Y_{\lambda}: \mathrm{d}\left(f_{\lambda}(x), g_{\lambda}(y)\right) \leq \varepsilon_{\lambda}\right\}$ and $s_{\lambda \lambda^{\prime}}^{\prime}(x, y)=\left(p_{\lambda \lambda^{\prime}}(x), q_{\lambda \lambda^{\prime}}(y)\right)$. It suffices to show that the system map $\boldsymbol{s}^{\prime}=\left(s_{\lambda}^{\prime}\right): E \rightarrow \boldsymbol{F}$ consisting of the maps $s_{\lambda}^{\prime}: E \rightarrow F_{\lambda}: s_{\lambda}^{\prime}(x, y)=s_{\lambda}(x, y)$ is a limit. For, if it is the case then $\boldsymbol{s}^{\prime}=\left(s_{\lambda}^{\prime}\right): E \rightarrow \boldsymbol{F}$ has properties (B1) and (B2) since each $F_{\lambda}$ is compact. That will imply that $s=\left(s_{\lambda}\right): E \rightarrow \boldsymbol{E}$ has properties (B1) and (B2). Suppose that $\boldsymbol{t}=\left(t_{\lambda}\right): E^{\prime} \rightarrow \boldsymbol{F}$ is a system map. We must find a unique map $\gamma: E^{\prime} \rightarrow E$ so that $\boldsymbol{t}=\boldsymbol{s}^{\prime} \gamma$. For each $e \in E^{\prime}$ and for each $\lambda \in \Lambda$ write $t_{\lambda}(e)=$ $\left(\alpha_{\lambda}(e), \beta_{\lambda}(e)\right) \in X_{\lambda} \times Y_{\lambda}$. Then the sets $\left\{\alpha_{\lambda}(e): \lambda \in \Lambda\right\}$ and $\left\{\beta_{\lambda}(e): \lambda \in \Lambda\right\}$ determine points $x_{e} \in X$ and $y_{e} \in Y$, respectively. The pair $\left(x_{e}, y_{e}\right)$ satisfies $\mathrm{d}\left(r_{\lambda} \varphi\left(x_{e}\right), r_{\lambda} \psi\left(y_{e}\right)\right)=\mathrm{d}\left(f_{\lambda} p_{\lambda}\left(x_{e}\right), g_{\lambda} q_{\lambda}\left(y_{e}\right)\right)=\mathrm{d}\left(f_{\lambda}\left(\alpha_{\lambda}(e)\right), g_{\lambda}\left(\beta_{\lambda}(e)\right)\right) \leq \varepsilon_{\lambda}$ for $\lambda \in \Lambda$. This together with property (AI) implies $r_{\lambda} f\left(x_{e}\right)=r_{\lambda} g\left(y_{e}\right)$ for $\lambda \in \Lambda$. So, $\varphi\left(x_{e}\right)=\psi\left(y_{e}\right)$. Thus the pair $\left(x_{e}, y_{e}\right)$ determines a point $\gamma(e) \in E$. We have a map $\gamma: E^{\prime} \rightarrow E: e \mapsto \gamma(e)$ such that $\boldsymbol{t}=\boldsymbol{s}^{\prime} \gamma$. That $\gamma$ is continuous follows from the fact that the functions $E^{\prime} \rightarrow X: e \mapsto x_{e}$ and $E^{\prime} \rightarrow Y: \quad e \mapsto y_{e}$ are continuous. The uniqueness of $\gamma$ follows from the fact that if $\gamma^{\prime}: E^{\prime} \rightarrow E$ is another map such that $t=s^{\prime} \gamma^{\prime}$, then $s_{\lambda}^{\prime} \gamma=s_{\lambda}^{\prime} \gamma^{\prime}$ for each $\lambda \in \Lambda$, so $\gamma=\gamma^{\prime}$.

TheOREM 9.2. For any pull-back in $\mathrm{CH}$

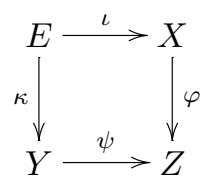

there is a commutative diagram in Apro- Top

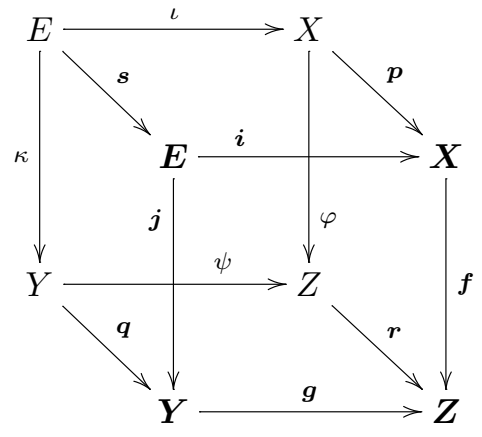


where the square

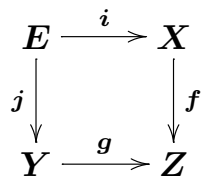

is a pull-back in Apro- ANR, and $\boldsymbol{p}: X \rightarrow \boldsymbol{X}, \boldsymbol{q}: Y \rightarrow \boldsymbol{Y}, \boldsymbol{r}: Z \rightarrow \boldsymbol{Z}$, and $s: E \rightarrow \boldsymbol{E}$ are ANR-resolutions.

Proof. By the argument in [7, Theorem 11] there exist systems of compact polyhedra $\boldsymbol{X}, \boldsymbol{Y}$, and $\boldsymbol{Z}$, resolutions $\boldsymbol{p}=\left(p_{\lambda}\right): X \rightarrow \boldsymbol{X}, \boldsymbol{q}=\left(q_{\mu}\right): Y \rightarrow$ $\boldsymbol{Y}$, and $\boldsymbol{r}=\left(r_{\nu}\right): \boldsymbol{Z} \rightarrow \boldsymbol{Z}$, system maps $\left(f, f_{\nu}\right): \boldsymbol{X} \rightarrow \boldsymbol{Z}$ and $\left(g, g_{\nu}\right): \boldsymbol{Y} \rightarrow \boldsymbol{Z}$ whose limits are $\varphi$ and $\psi$, respectively. By Lemma 7.1 and Proposition 2.7 we can assume that $\boldsymbol{X}, \boldsymbol{Y}$, and $\boldsymbol{Z}$ have the same index set $\Lambda$, the system maps are level maps $\left(f_{\lambda}\right)$ and $\left(g_{\lambda}\right)$, and there is a commutative approximate system $\mathfrak{Z}=\left(Z_{\lambda}, \varepsilon_{\lambda}, r_{\lambda \lambda^{\prime}}, \Lambda\right)$ associated with $\boldsymbol{Z}$. Let $\boldsymbol{f}: \boldsymbol{X} \rightarrow \boldsymbol{Z}$ and $\boldsymbol{g}: \boldsymbol{Y} \rightarrow \boldsymbol{Z}$ be the morphisms in Apro- ANR represented by $\left(f_{\lambda}\right)$ and $\left(g_{\lambda}\right)$, respectively. Consider the pull-back in Apro- ANR:

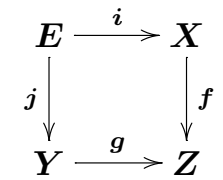

where $\boldsymbol{E}=\left(E_{\lambda}, s_{\lambda \lambda^{\prime}}, \Lambda\right)$ is the ANR-system such that

$$
E_{\lambda}=\left\{(x, y) \in X_{\lambda} \times Y_{\lambda}: \mathrm{d}\left(f_{\lambda}(x), g_{\lambda}(y)\right)<\varepsilon_{\lambda}\right\}
$$

and $s_{\lambda \lambda^{\prime}}: E_{\lambda^{\prime}} \rightarrow E_{\lambda}$ is the map defined by $s_{\lambda \lambda^{\prime}}(x, y)=\left(p_{\lambda \lambda^{\prime}}(x), q_{\lambda \lambda^{\prime}}(y)\right)$. Then the system map $s=\left(s_{\lambda}\right): E \rightarrow \boldsymbol{E}$ defined by $s_{\lambda}(x, y)=\left(p_{\lambda}(x), q_{\lambda}(y)\right)$ is an ANR-resolution by the same argument as in the proof of Theorem 9.1. This proves the theorem.

\section{REFERENCES}

[1] M. Artin and B. Mazur, Etale homotopy, Lecture Notes in Mathematics 100, Springer-Verlag, Berlin-New York, 1969.

[2] H. J. Baues, Algebraic homotopy, Cambridge Studies in Advanced Mathematics 15, Cambridge University Press, Cambridge, 1989.

[3] Q. Haxhibeqiri, Shape fibration for topological spaces, Glas. Mat. Ser. III 17(37) (1982), 381-401.

[4] Q. Haxhibeqiri, The exact sequence of a shape fibration, Glas. Mat. Ser. III 18(38) (1983), 157-177.

[5] J. R. Isbell, Uniform spaces, Mathematical Surveys 12, American Mathematical Society, Providence, R.I., 1964.

[6] M. Jani, Induced shape fibrations and fiber shape equivalence, Rocky Mountain J. Math. 12 (1982), 305-332.

[7] S. Mardešić, Approximate polyhedra, resolutions of maps and shape fibrations, Fund. Math. 114 (1981), 53-78. 
[8] S. Mardešić and T. B. Rushing, Shape fibrations. I, General Topology Appl. 9 (1978), 193-215.

[9] S. Mardešić and J. Segal, Shape Theory. The inverse system approach, North-Holland Mathematical Library 26, North-Holland Publishing Co., Amsterdam-New York, 1982.

[10] S. Mardešić and T. Watanabe, Approximate resolutions of spaces and mappings, Glas. Mat. Ser. III 24(44) (1989), 587-637.

[11] C. R. F. Maunder, Algebraic Topology, Van Nostrand Reinhold Company, 1970.

[12] T. Miyata, Uniform shape theory, Glas. Mat. Ser. III 29(49) (1994), 123-168.

[13] T. Miyata, Fibrations in the category of absolute neighborhood retracts, Bull. Pol. Acad. Sci. Math. 55 (2007), 145-154.

[14] T. Miyata, Strong pro-fibrations and ANR objects in pro-categories, mimeographic note, Department of Mathematics and Informatics, Kobe University.

[15] T. Miyata, On the pro-categories of abelian categories, mimeographic note, Department of Mathematics and Informatics, Kobe University.

[16] T. Miyata and T. Watanabe, Approximate resolutions of uniform spaces, Topology Appl. 113 (2001), 211-241.

[17] T. Watanabe, Approximative shape. I. Basic notions, Tsukuba J. Math. 11 (1987), 17-59.

T. Miyata

Department of Mathematics and Informatics

Graduate School of Human Development and Environment

Kobe University

Kobe, 657-8501

Japan

E-mail: tmiyata@kobe-u.ac.jp

Received: $20.8 \cdot 2007$.

Revised: 21.12.2007. 